\title{
AMENABLE COVERS, VOLUME AND L L'BETTI NUMBERS OF ASPHERICAL MANIFOLDS
}

\author{
ROMAN SAUER
}

\begin{abstract}
We provide a proof for an inequality between volume and $\mathrm{L}^{2}$ Betti numbers of aspherical manifolds for which Gromov outlined a strategy based on general ideas of Connes. The implementation of that strategy involves measured equivalence relations, Gaboriau's theory of $L^{2}$-Betti numbers of $\mathscr{R}$-simplicial complexes, and other themes of measurable group theory. Further, we prove new vanishing theorems for $L^{2}$-Betti numbers that generalize a classical result of Cheeger and Gromov. As one of the corollaries, we obtain a gap theorem which implies vanishing of $L^{2}$-Betti numbers of an aspherical manifold when its minimal volume is sufficiently small.
\end{abstract}

\section{Contents}

1. Introduction

1.1. Discussion of results

1.2. Conventions

1.3. On the approach

2. $\mathscr{R}$-Spaces and $\mathscr{R}$-simplicial Complexes

2.1. $\mathscr{R}$-simplicial complexes

2.2. Standard embeddings

2.3. $\mathscr{R}$-spaces and geometric $\mathscr{R}$-maps

2.4. Simplicial approximation of geometric $\mathscr{R}$-maps 10

2.5. $\mathscr{R}$-covers

3. Singular foliated homology

3.2. Singular homology of $\mathscr{R}$-spaces

3.3. Functoriality and homotopy invariance 18

$\begin{array}{ll}3.4 . & \text { Support mass } \\ 3 & 19\end{array}$

3.5. Fundamental classes $\quad 22$

4. Proof of Theorem A 23

4.1. $\mathscr{R}$-covers with controlled multiplicity 23

4.2. The map to the nerve 24

4.3. Conclusion of proof 28

5. Proofs of Theorems B and C 29

5.1. $\mathscr{R}$-covers from the Ornstein-Weiss-Rokhlin lemma 29

5.2. Estimating the number of $\mathscr{R}$-cells of the nerve $\mathscr{N}(\mathscr{U} \delta)$

5.3. Conclusion of proofs 33

Appendix A. $\mathrm{L}^{2}$-Betti numbers and the support mass of the fundamental class $\quad 34$

References $\quad 36$

1991 Mathematics Subject Classification. Primary: 22D20,53C20,58J22; Secondary: 20F32,57N65.

The author thanks Clara Löh for corrections and suggestions for improving the manuscript. He acknowledges support of the German science foundation (DFG), made through grant SA 1661/1-1. 


\section{INTRODUCTION}

1.1. Discussion of results. The minimal volume minvol $(M)$ of a smooth manifold $M$ is the infimum of volumes of complete Riemannian metrics on $M$ whose sectional curvature is pinched between -1 and 1 . Questions of significant geometric interest are: Is $\operatorname{minvol}(M)>0$ ? If yes, is the infimum attained by a metric of $M$ ? If yes, is this metric unique, or does it satisfy certain regularity properties? In the seminal paper [19] Gromov introduced the notion of simplicial volume and initiated the use of simplicial volume and bounded cohomology as basic tools in the study of the minimal volume. The central fact is that the simplicial volume provides a homotopy invariant that bounds the minimal volume from below.

In the present paper we transfer classical vanishing theorems for simplicial volume and bounded cohomology and theorems relating volume and simplicial volume of manifolds to $L^{2}$-Betti numbers of closed, aspherical manifolds or spaces.

A manifold $M$ is called aspherical if its universal covering is contractible or, equivalently, $M$ is a model of the classifying space $B \pi_{1}(M)$ of its fundamental group. $L^{2}$-Betti numbers for regular coverings of closed Riemannian manifolds were introduced by Atiyah [1], and their range of definition and application was widened over the years $[7,10-12,26]$. By now there is a definition of $L^{2}$-Betti numbers $b_{i}^{(2)}(Y ; \Gamma) \in[0, \infty], i \geq 0$, of an arbitrary space $Y$ with the action of a discrete group $\Gamma[27$, chapter 6$]$. The most important case for us is the universal covering $\widetilde{M}$ of a space $M$ with the natural action of its fundamental group $\pi_{1}(M)$. Here we omit $\pi_{1}(M)$ in the notation and simply write $b_{i}^{(2)}(\widetilde{M})$.

We provide a proof (Section 4) of the following inequality stated by Gromov in [23, Section 5.33 on p. 297], where he outlines a strategy based on general ideas of Connes. A major part of this paper deals with a rigorous implementation of this strategy, which takes a considerable effort. See Subsection 1.3 for an overview.

Theorem A. Let $C>0$ and $n \in \mathbb{N}$. Then there is a constant const $_{C, n}>0$ with the following property: If $M$ is an $n$-dimensional, closed, aspherical Riemannian manifold such that its universal covering $\widetilde{M}$ with the induced metric has the property (packing inequality) that each ball of radius 1 contains at most $\mathrm{Cr}^{-n}$ disjoint balls of radius $r$ for every $0<r<1$, then

$$
b_{i}^{(2)}(\widetilde{M}) \leq \operatorname{const}_{C, n} \operatorname{vol}(M) \text { for all } i \geq 0 .
$$

Let us briefly recall the well known relation between a lower Ricci curvature bound and a packing inequality like in the hypothesis of the preceding theorem. If $M$ satisfies the lower Ricci curvature bound $\operatorname{Ricci}(M) \geq-(n-1)$ then also $\operatorname{Ricci}(\widetilde{M}) \geq-(n-1)$. Write $B(m, r) \subset \widetilde{M}$ for the ball of radius $r$ around $m \in \widetilde{M}$, and $B_{h y p}(r)$ for a ball of radius $r$ in hyperbolic $n$-space. According to the BishopGromov inequality [18, Theorem 4.19], if $R>r$, then

$$
\frac{\operatorname{vol}(B(m, R))}{\operatorname{vol}(B(m, r))} \leq \frac{\operatorname{vol}\left(B_{h y p}(R)\right)}{\operatorname{vol}\left(B_{\text {hyp }}(r)\right)} .
$$

Since the right hand side can bounded by $\operatorname{const}_{n}(R / r)^{n}$ for $r, R \leq 1$, a mere volume estimate implies that at most const $_{n} r^{-n}$ disjoint balls of radius $r$ fit into a ball of radius 1 for a constant const $_{n}$ only depending on the dimension $n$. Thus the following corollaries are direct consequences of Theorem A.

Corollary. For each $n \in \mathbb{N}$ there is a constant const $_{n}>0$ with the following property: If $M$ is an $n$-dimensional, closed, aspherical Riemannian manifold with lower Ricci curvature bound $\operatorname{Ricci}(M) \geq-(n-1)$, then

$$
b_{i}^{(2)}(\widetilde{M}) \leq \operatorname{const}_{n} \operatorname{vol}(M) \text { for all } i \geq 0 .
$$


Corollary (Main inequality for $L^{2}$-Betti numbers). For each $n \in \mathbb{N}$ there is a

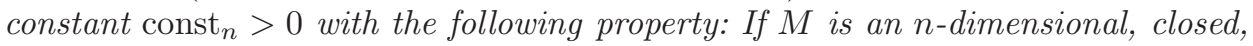
aspherical manifold $M$, then

$$
b_{i}^{(2)}(\widetilde{M}) \leq \operatorname{const}_{n} \operatorname{minvol}(M) \text { for all } i \geq 0 .
$$

These two corollaries are the analog of Gromov's main inequality [19, 0.5] with simplicial volume replaced by $L^{2}$-Betti numbers and the additional hypothesis of asphericity. However, Gromov's main inequality also applies to non-compact complete manifolds, and the constant is explicitly known.

Notice that for aspherical $M$ we have $b_{i}^{(2)}(\widetilde{M})=b_{i}^{(2)}\left(\pi_{1}(M)\right)$, and $b_{i}^{(2)}\left(\pi_{1}(M)\right)$ is an orbit equivalence invariant of the fundamental group $\pi_{1}(M)$ by Gaboriau's work [17]. It is a particularly interesting aspect of the previous corollary that it provides a non-trivial orbit equivalence invariant that bounds the minimal volume from below.

According to the Hopf-Singer conjecture [27, Conjecture 11.1]), $b_{i}^{(2)}(\widetilde{M})=0$ if $2 i \neq \operatorname{dim}(M)$ for every closed, aspherical manifold $M$. Examples of closed aspherical even-dimensional manifolds where the $L^{2}$-Betti number in the middle dimension is positive and the Hopf-Singer conjecture holds true include Kähler hyperbolic manifolds [21] and closed locally symmetric spaces of fundamental rank zero [5].

We prove the following new vanishing Theorem in Section 5. Recall that a subset $U \subset M$ of a topological space is amenable if for every $x \in U$ the image $\operatorname{im}\left(\pi_{1}(U ; x) \rightarrow \pi_{1}(M ; x)\right)$ is amenable.

Theorem B. Let $M$ be an n-dimensional, closed, triangulated (e.g. smooth), aspherical manifold. Assume that $M$ is covered by open, amenable sets such that every point belongs to at most $n$ sets. Then

$$
b_{i}^{(2)}(\widetilde{M})=0 \text { for all } i \geq 0 .
$$

Note that the original definitions of $L^{2}$-Betti numbers of Atiyah and Dodziuk do not apply to topological manifolds without triangulations, but Lück's theory does. Theorem B actually stays true without the assumption triangulated but we omit the necessary modifications in this case due to the length of the paper.

We remark that a naive spectral sequence approach to the theorem above fails since the amenable subsets are in general not aspherical, thus may have nonvanishing $L^{2}$-cohomology.

The following result for simplicial complexes is also obtained in Section 5.

Theorem C. Let $M$ be a finite aspherical simplicial complex. Assume that $M$ is covered by open amenable sets such that every point belongs to at most $n$ subsets. Then

$$
b_{i}^{(2)}(\widetilde{M})=0 \text { for all } i \geq n .
$$

Theorems B and C together are the analog of Gromov's vanishing theorem [19, Section 3.1] for $L^{2}$-Betti numbers. Furthermore, Theorem $\mathrm{C}$ generalizes a classical theorem of Cheeger and Gromov [7], which says that the $L^{2}$-Betti numbers of aspherical simplicial complexes with amenable fundamental group vanish.

Gromov shows [19, Section 3.4] that for every dimension $n \in \mathbb{N}$ there is a constant $\epsilon_{n}>0$ with the property: If $M$ is an $n$-dimensional, closed Riemannian manifold such that $\operatorname{Ricci}(M) \geq-(n-1)$ and $\operatorname{vol}(B(1)) \leq \epsilon_{n}$ for each unit ball $B(1) \subset M$ then there is an amenable cover as in the hypothesis of Theorem B; his proof can be simplified a bit using the more recent Margulis lemma for Ricci curvature by Cheeger and Colding [8, Theorem 8.7; 19, Section 3.4]. Hence we obtain the following interesting gap theorems. 
Corollary. For every $n \in \mathbb{N}$ there is a constant $\epsilon_{n}>0$ with the following property: If $M$ is an $n$-dimensional, closed, aspherical Riemannian manifold such that $\operatorname{Ricci}(M) \geq-(n-1)$ and $\operatorname{vol}(B(1)) \leq \epsilon_{n}$ for each unit ball $B(1) \subset M$ then

$$
b_{i}^{(2)}(\widetilde{M})=0 \text { for all } i \geq 0 \text {. }
$$

Corollary (Isolation theorem for $L^{2}$-Betti numbers). For every $n \in \mathbb{N}$ there is a constant $\epsilon_{n}>0$ with the following property: If $M$ is an n-dimensional, closed, aspherical, smooth manifold such that $\operatorname{minvol}(M) \leq \epsilon_{n}$, then

$$
b_{i}^{(2)}(\widetilde{M})=0 \text { for } i \geq 0 .
$$

This is the analog of Gromov's isolation theorem [19, Section 0.5] for $L^{2}$-Betti numbers.

The Atiyah conjecture for a group $\Gamma$ [27, Chapter 10] predicts that the $L^{2}$-Betti numbers of any closed aspherical manifold with fundamental group $\Gamma$ are integers. Provided the Atiyah conjecture holds true, the previous corollaries would follow from Theorem A and its corollaries. So far the Atiyah conjecture has been verified for certain inductively defined classes of groups but not for fundamental groups of manifolds satisfying the hypothesis of the corollaries above nor for any geometrically defined class of groups.

As mentioned above, all stated results are analogs of theorems about simplicial volume or bounded cohomology in Gromov's paper [19]. Unlike there, the hypothesis of asphericity is essential in the present context. For example, Theorems A and B both fail for the 3 -sphere, which has vanishing minimal volume.

Theorems A and B would follow from Gromov's results [19] if the following conjecture, which Gromov formulated as a question, would be true [22, Section 8A on p. 232; 23, Remark e) on p. 304; 27, Chapter 14].

Conjecture. For every dimension $n \in \mathbb{N}$ there is a constant const ${ }_{n}>0$ such that for every $n$-dimensional, closed, aspherical, orientable manifold $M$ we have

$$
b_{i}^{(2)}(\widetilde{M}) \leq \text { const }_{n}\|M\| \text { for all } i \geq 0 .
$$

Here $\|M\|$ denotes the simplicial volume of $M$.

1.2. Conventions. The following framework is used throughout the paper.

Assumption 1.1. Let $(X, \mu)$ be a standard Borel space equipped with an atomfree probability Borel measure $\mu$. Let $\Gamma$ be a countable group acting (essentially) freely and $\mu$-preservingly on $X$. The orbit equivalence relation $\mathscr{R}$ is the equivalence relation on $X$ given by

$$
\mathscr{R}=\{(\gamma x, x) \in X \times X ; \gamma \in \Gamma, x \in X\} .
$$

Here the $\Gamma$-action is essentially free if $X_{0}=\left\{x \in X ; \Gamma_{x} \neq\{1\}\right\}$ is a $\mu$-null set. Upon replacing $X$ with $X \backslash X_{0}$, we can always achieve that the action is strictly free. Therefore the word essentially will be frequently omitted. Recall that $(X, \mu)$ as a measure space is isomorphic to $([0,1], \lambda)$ where $\lambda$ denotes the Lebesgue measure.

An orbit equivalence relation is an example of a countable measured equivalence relation. For more information about that notion we refer to $[13 ; 16$, Section 2 ; 17, Section 0]. Every countable measured equivalence relation arises as an orbit equivalence relation of a (not necessarily free) action of a countable group.

For later reference, we record the following assumptions.

Assumption 1.2. Let $M$ be a connected, finite simplicial complex with fundamental group $\Gamma$. Let $(X, \mu)$ and $\mathscr{R}$ be as in Assumption 1.1. All metric notions about $M$ refer to the unique length metric that restricts to the standard Euclidean metric on simplices. 
Assumption 1.3. Let $M$ be an $n$-dimensional, connected, closed, oriented, triangulated manifold with fundamental group $\Gamma$. Let $(X, \mu)$ and $\mathscr{R}$ be as in Assumption 1.1.

Without loss of generality, we assume for the proofs of Theorems A and B that the manifold $M$ is connected and orientable, that is, satisfies Assumption 1.3. If $M$ is non-orientable then there is a two-fold orientation cover $p: \bar{M} \rightarrow M$. If $M$ satisfies the hypothesis of Theorem A, then $\bar{M}$ with the induced Riemannian metric does so. If $M$ has an amenable cover $\left\{U_{i}\right\}_{i \in I}$ as in the hypothesis of Theorem B, then $\left\{p^{-1}(\bar{M})\right\}_{i \in I}$ is one for $\bar{M}$. Because of multiplicativity [27, Theorem $\left.1.35(9)\right]$

$$
b_{i}^{(2)}(\widetilde{\bar{M}})=2 b_{i}^{(2)}(\widetilde{M}) \text { for all } i \geq 0
$$

it is sufficient to prove the theorem for $\bar{M}$.

A countable family $\left(X_{i}\right)_{i \in I}$ of Borel subsets of a measure space $(X, \mu)$ is called a (countable) Borel partition if $\bigcup_{i \in I} X_{i}$ is a $\mu$-conull set and $\mu\left(X_{i} \cap X_{j}\right)=0$ for $i \neq j$. By abuse of notation, we just write $X=\bigcup_{i \in I} X_{i}$. The abbreviation a.e. means either almost every or almost everywhere.

1.3. On the approach. We present an elaborate version of Gromov's strategy [23, Section 5.33] to attack Theorem A, which itself is motivated by general ideas of Connes. The appropriate framework involves techniques from measured equivalence relations, Connes' and Gaboriau's theory of $\mathscr{R}$-simplicial complexes and other themes of measurable group theory [36].

The way we set up the general framework is flexible enough to run the proofs of Theorems B and C. However, a crucial difference is the geometric construction of $\mathscr{R}$-covers which involves the Ornstein-Weiss Lemma.

1.3.1. General Remark on bounding $\left(L^{2}\right)$-Betti numbers. One method to bound the $i$-th Betti number and the $i$-th $L^{2}$-Betti number of a topological space $M$ from above by a constant $C$ is to realize $M$ as a homotopy retract in a simplicial complex $S$ (that is, there are maps $f: M \rightarrow S$ and $g: S \rightarrow M$ with $g \circ f \simeq \operatorname{id}_{M}$ ) such that the number of $i$-simplices of $S$ is at most $C$.

In the sequel let $M$ be as in Assumption 1.3, and assume that $M$ is aspherical, that is, $\widetilde{M}$ is model of $E \Gamma$, Then one could alternatively try to find a free $\Gamma$-simplicial complex $S$ with at most $C$ equivariant $i$-simplices and an equivariant map $f: \widetilde{M} \rightarrow$ $S$. The advantage of asphericity and working equivariantly is that one automatically gets an equivariant map $g: S \rightarrow \widetilde{M}$ and an equivariant homotopy $g \circ f \simeq \operatorname{id}_{\widetilde{M}}$ from the universal property of $E \Gamma$, which then leads to the same estimate.

1.3.2. The category of $\mathscr{R}$-spaces. We use a similar method (which does not work anymore to bound Betti numbers) in the category of $\mathscr{R}$-spaces (Section 2 ) instead of $\Gamma$-spaces, where $\mathscr{R}$ is as in Assumption 1.1. An $\mathscr{R}$-space is the realization of a fiberwise locally finite $\mathscr{R}$-simplicial complex (Definition 2.6) in the sense of $[17$, Section 2]. One example is $X \times \widetilde{M}$ with the $\mathscr{R}$-action $(\gamma x, x) \cdot(x, m)=(\gamma x, \gamma m)$. The morphism in this category, called geometric $\mathscr{R}$-maps (Definition 2.21), are fiberwise continuous and proper. The category of $\mathscr{R}$-spaces is an extension of the combinatorial framework [17] to a topological one.

1.3.3. Homotopy retracts of $\mathscr{R}$-spaces by $\mathscr{R}$-covers. An $\mathscr{R}$-cover $\mathscr{U}$ of $X \times \widetilde{M}$ is an equivariant family of sets of the form $A \times U$ with $A \subset X$ Borel and $U \subset \widetilde{M}$ open (Definition 2.27). Such an $\mathscr{R}$-cover gives rise to a nerve construction $\mathscr{N}(\mathscr{U})$, which is naturally an $\mathscr{R}$-simplicial complex, and a geometric $\mathscr{R}$-map

$$
\phi: X \times \widetilde{M} \rightarrow \mathscr{N}(\mathscr{U})
$$


Also in this context asphericity yields a homotopy retract, that is, a geometric $\mathscr{R}$-map $\psi: \mathscr{N}(\mathscr{U}) \rightarrow X \times \widetilde{M}$ with $\psi \circ \phi \simeq \operatorname{id}_{X \times \widetilde{M}}$.

1.3.4. Bounding the $L^{2}$-Betti number in the top dimension $n$ under the hypothesis of Theorem A. Now let $M$ be as in Theorem A. It is a standard trick that one obtains from the packing type hypothesis on $\widetilde{M}$ a cover whose multiplicity is bounded in terms of the constant $C$ [4, Lemma 125 on p. 333]. But in general there is no way to make such a cover $\Gamma$-equivariant without loosing control over its multiplicity. If there would be, we could prove Theorem A by proceeding as in 1.3.1.

However, it is possible to construct an $\mathscr{R}$-cover $\mathscr{U}$ such that the induced cover on $\{x\} \times \widetilde{M}$ has the correct multiplicity for a.e. $x \in X$ (Theorem 4.1). Then one can modify $\phi$ from (1.1) by a geometric $\mathscr{R}$-homotopy such that the equivariant number of $n$-simplices of $\operatorname{im}(\phi) \subset \mathscr{N}(\mathscr{U})$ in the sense of Definition 2.9 (equivalently: the measure $\nu^{n}\left(\mathscr{R} \backslash \Sigma^{(n)}\right)$ defined in [17, Section 2.2.3]) is dominated by $\operatorname{const}_{C, n} \operatorname{vol}(M)$ where const $_{C, n}$ is a constant only depending on the dimension $n$ and the constant $C$ from Theorem A.

Now an application of Gaboriau's theory [17] would yield the stated bound

$$
b_{n}^{(2)}(\widetilde{M}) \leq \operatorname{const}_{C, n} \operatorname{vol}(M)
$$

in the top dimension, which alone is useless if one assumes the Hopf-Singer conjecture for $M$ (mentioned in Subsection 1.1). We introduce another tool to obtain the bound in all degrees: Foliated singular homology and the support mass.

1.3.5. Foliated singular homology. We define a homology theory $\mathscr{H}_{*}$ (Section 3 ) for the category of $\mathscr{R}$-spaces, called foliated singular homology that is a singular version of the sheaf-theoretic tangential homology of measured foliations [29].

Actually, $\mathscr{H}_{*}(\Sigma)$ for an $\mathscr{R}$-space $\Sigma$ is really defined in terms of the laminated quotient space $\mathscr{R} \backslash \Sigma$, but for conceptual and technical reasons it is better to work with the $\mathscr{R}$-space instead of its quotient. For example, the universal property that gives us the map $\psi$ is not transparent on the quotient level.

The definition of $\mathscr{H}_{n}(\Sigma)$ is modelled on Gromov's description of Connes's foliated simplicial volume [20, 2.4.B]. Indeed, the foliated simplicial volume for the measurable foliation $\Gamma \backslash(X \times \widetilde{M})$ is most naturally defined in terms of cycles representing the fundamental class in $\mathscr{H}_{n}(X \times \widetilde{M})$ (see Remark 3.26 for the notion of fundamental class). For our purposes, we deal with another, yet related numerical invariant on $\mathscr{H}_{n}(\Sigma)$, called the support mass and defined in Subsection 3.4.

It turns out that in the situation of 1.3 .4 we not only get the bound (1.2), but, using the properties of $\mathscr{H}_{*}$ developed in Section 3 , we can also bound the support mass of the fundamental class in $\mathscr{H}_{n}(X \times \widetilde{M})$ by const $_{C, n} \operatorname{vol}(M)$ (Theorem 4.3). This implies (Theorem 3.25) that the image of the fundamental class in $\mathrm{H}_{n}(M ; \mathbb{Z})$ under the homomorphism induced by inclusion of coefficients

$\mathrm{H}_{n}(M ; \mathbb{Z})=\mathrm{H}_{n}\left(\mathbb{Z} \otimes_{\mathbb{Z} \Gamma} \mathrm{C}_{*}(\widetilde{M})\right) \rightarrow \mathrm{H}_{n}\left(\mathrm{~L}^{\infty}(X ; \mathbb{Z}) \otimes_{\mathbb{Z} \Gamma} \mathrm{C}_{*}(\widetilde{M})\right)=\mathrm{H}_{n}^{\Gamma}\left(\widetilde{M} ; \mathrm{L}^{\infty}(X ; \mathbb{Z})\right)$

can be represented by a cycle $\sum_{i=1}^{m} f_{i} \otimes \sigma_{i}$ with $f_{i} \in \mathrm{L}^{\infty}(X ; \mathbb{Z})$ and $\sigma_{i} \in \operatorname{Sing}_{n}(\widetilde{M})$ such that

$$
\sum_{i=1}^{m} \mu\left(\operatorname{supp}\left(f_{i}\right)\right)<\operatorname{const}_{C, n} \operatorname{vol}(M) \text {. }
$$

Such a representing cycle of the fundamental class leads to an estimate for $b_{i}^{(2)}(\widetilde{M})$ for all $i \geq 0$ (in our case: $b_{i}^{(2)}(\widetilde{M}) \leq \operatorname{const}_{C, n} \operatorname{vol}(M)$ ) by a Poincaré duality argument. See Theorem A.1 in the Appendix. 
1.3.6. The $\mathscr{R}$-covers used for Theorems $B$ and $C$. The proofs of Theorems $\mathrm{B}$ and $\mathrm{C}$ differ from that of Theorem $\mathrm{A}$ in the construction of the $\mathscr{R}$-covers $\mathscr{U}$. Whereas in the case of Theorem A the input from ergodic theory is very modest, we now employ the generalized Rokhlin lemma of Ornstein and Weiss [32] to construct suitable $\mathscr{R}$ covers $\mathscr{U}_{\delta}$ for any $\delta>0$ such that the number of equivariant $k$-simplices of $\mathscr{N}(\mathscr{U})$ is less than const $\delta$ for $k \geq n$. The characteristic feature of elements $A \times U$ of $\mathscr{U}_{\delta}$, where $A \subset X$ and $U \subset \widetilde{M}$, is that $\mu(A)$ gets small and $U$ gets large when $\delta$ tends to zero.

The proof of Theorem $\mathrm{C}$ is easier than that of Theorem B since we do not need the technique in 1.3.5. Instead we apply Gaboriau's techniques [17].

\section{2. $\mathscr{R}$-SpaCes AND $\mathscr{R}$-Simplicial Complexes}

In Subsections 2.1 to $2.4, \mathscr{R}$ denotes an arbitrary countable measured equivalence relation on a standard Borel space $(X, \mu)$ equipped with a probability Borel measure $\mu$. In Subsection 2.5 we refer to the more special situation of Assumption 1.1.

2.1. $\mathscr{R}$-simplicial complexes. To fix the terminology and for the convenience of the reader, we recall in Subsection 2.1 basic notions about $\mathscr{R}$-simplicial complexes as presented in [17].

Definition 2.1. An $(X, \mu)$-space or $X$-space is a standard Borel space $\Sigma$ together with a Borel map $p_{\Sigma}: \Sigma \rightarrow X$ such that the fibers $\Sigma_{x}=p_{\Sigma}^{-1}(x), x \in X$, are countable. A map of $X$-spaces $f: \Sigma \rightarrow \Phi$ is a Borel map such that $\operatorname{pr}_{\Phi} \circ f=\operatorname{pr}_{\Sigma}$.

We denote the fiber product of $X$-spaces $\Sigma \times_{X} \Sigma^{\prime}=\left\{(u, v) \in \Sigma \times \Sigma^{\prime} ; p_{\Sigma}(u)=\right.$ $\left.p_{\Sigma^{\prime}}(v)\right\}$ by $\Sigma * \Sigma^{\prime}$.

Definition 2.2. An $\mathscr{R}$-action on an $X$-space $\Sigma \rightarrow X$ is map of $X$-spaces $\mathscr{R} * \Sigma \rightarrow \Sigma$, $((y, x), u) \mapsto(y, x) . u$, such that

a) $(y, x) \cdot \Sigma_{x} \subset \Sigma_{y}$,

b) $(x, x) . u=u$ for every $x \in X$ and every $u \in \Sigma_{x}$, and

c) $(z, y) \cdot((y, x) \cdot u)=(z, x) \cdot u$ for all $x, y, z \in X$ and every $u \in \Sigma_{x}$.

Remark 2.3. If $\mathscr{R}$ is as in Assumption 1.1, then an $\mathscr{R}$-action on $\Sigma$ is nothing else but a measurable $\Gamma$-action on $\Sigma$ such that $\gamma \cdot \Sigma_{x} \subset \Sigma_{\gamma x}$.

Fibered products of $X$-spaces with $\mathscr{R}$-actions carry by definition the diagonal $\mathscr{R}$-action. An $\mathscr{R}$-action that has a Borel fundamental domain $\mathscr{F}$, that is a Borel subset of $\Sigma$ whose intersection with every $\mathscr{R}$-orbit consists of exactly one element, is called discrete.

Definition 2.4. The natural measure on the $(X, \mu)$-space $\Sigma$ is defined as

$$
\nu^{\mu}(U)=\int_{X} \#\left(p_{\Sigma}^{-1}(x) \cap U\right) d \mu(x) \text { for all } U \subset \Sigma \text { Borel. }
$$

If the choice of $\mu$ is clear from the context, the superscript $\mu$ in $\nu^{\mu}(U)$ is omitted.

Definition 2.5. Let $\Sigma$ be an $(X, \mu)$-space with a discrete $\mathscr{R}$-action. The measure $\nu_{t}^{\mu}$ on $\mathscr{R} \backslash \Sigma$ obtained from the restriction $\left.\nu^{\mu}\right|_{\mathscr{F}}$ of the natural measure to a Borel fundamental domain $\mathscr{F} \subset \Sigma$ after identifying $\mathscr{R} \backslash \Sigma$ with $\mathscr{F}$ is called the transversal measure on $\mathscr{R} \backslash \Sigma$. As before, the superscript $\mu$ is omitted in the notation if the choice of $\mu$ is clear from the context.

Remark. It is easily verified that the preceding definition does not depend on the choice of the Borel fundamental domain.

Definition 2.6. An $\mathscr{R}$-simplicial complex $\Sigma$ consists of the following data: - an $X$-space $\Sigma^{(0)} \rightarrow X$ with a discrete $\mathscr{R}$-action. 
- for each $n \in \mathbb{N}$ a Borel subset $\Sigma^{(n)} \subset \underbrace{\Sigma^{(0)} * \Sigma^{(0)} * \ldots * \Sigma^{(0)}}_{n+1 \text { times }}$ (ordered $n$-simplices)

subject to four conditions:

a) (permutations) $\Sigma^{(n)}$ is invariant under permutations of the coordinates.

b) (non-degeneracy) $\left(v_{0}, \ldots, v_{n}\right) \in \Sigma^{(n)}$ implies $v_{0} \neq v_{n}$.

c) (boundary condition) $\left(v_{0}, \ldots, v_{n}\right) \in \Sigma^{(n)}$ implies $\left(v_{1}, \ldots, v_{n}\right) \in \Sigma^{(n-1)}$.

d) (invariance) $\mathscr{R} \cdot \Sigma^{(n)}=\Sigma^{(n)}$.

Definition 2.7. An $\mathscr{R}$-simplicial map $\phi: \Sigma \rightarrow \Phi$ between $\mathscr{R}$-simplicial complexes is a Borel map over $X$ such that $\phi_{x}: \Sigma_{x} \rightarrow \Phi_{x}$ is simplicial for a.e. $x \in X$ and $\phi$ is $\mathscr{R}$-equivariant in the sense that for a.e. $x \in X$, every $y \in X$ with $(y, x) \in \mathscr{R}$, and every $m \in \Sigma_{x}$ we have $\phi((y, x) . m)=(y, x) . \phi(m)$.

The reader may notice that while the notion of an $\mathscr{R}$-action (Definition 2.2) is defined in a strict sense the conditions of the previous definition are only required up to null-sets.

Definition 2.8. The barycentric subdivision $\operatorname{sd}(\Sigma)$ of $\Sigma$ is the $\mathscr{R}$-simplicial complex obtained by taking the barycentric subdivision on each fiber $\Sigma_{x}$. The $n$-fold barycentric subdivision is denoted by $\operatorname{sd}^{(n)}(\Sigma)$. Abstractly, $\operatorname{sd}(\Sigma)$ is defined as follows. There is a natural action of the symmetric group $\mathbf{S}(n+1)$ on $\Sigma^{(n)}$ which commutes with the $\mathscr{R}$-action. The quotient $\mathbf{S}(n+1) \backslash \Sigma^{(n)}$ is still an $X$-space with a discrete $\mathscr{R}$-action. The set of 0 -simplices is

$$
\operatorname{sd}(\Sigma)^{(0)}=\coprod_{n \geq 0} \mathbf{S}(n+1) \backslash \Sigma^{(n)} .
$$

Thus $\operatorname{sd}(\Sigma)^{(0)}$ is partially ordered by inclusion. The set of $n$-simplices $\operatorname{sd}(\Sigma)^{(n)}$ consists of $(n+1)$-tuples $\left(v_{0}, \ldots, v_{n}\right)$ with the property that the $v_{i}$ lie in some common fiber of $\operatorname{sd}(\Sigma)^{(0)}$ and $\left\{v_{0}, \ldots, v_{n}\right\}$ can be totally ordered.

Definition 2.9. The (weighted) number of (non-oriented) equivariant $n$-simplices of an $\mathscr{R}$-simplicial complex $\Sigma$ is defined as $\nu_{t}\left((\mathbf{S}(n+1) \times \mathscr{R}) \backslash \Sigma^{(n)}\right)$ and denoted by $\sigma_{n}(\Sigma)$.

Example 2.10. Retain the notation of Assumption 1.1. Let $S$ be a free $\Gamma$-equivariant simplicial complex Then $X \times S$ is an $\mathscr{R}$-simplicial complex via

$$
(\gamma x, x)(x, s)=(\gamma x, \gamma s)
$$

The set of $n$-simplices is $\Sigma^{(n)}=X \times S^{(n)}$.

Since an $\mathscr{R}$-action is defined in a strict sense, we may and will assume here that the $\Gamma$-action on $X$ is strictly free (cf. Subsection 1.2).

At any rate, in the proofs of Theorems A, B and $\mathrm{C}$ we fix the group $\Gamma$ and thus could also speak of $\Gamma$ - instead of $\mathscr{R}$-actions (cf. Remark 2.3), but the framework of $\mathscr{R}$-actions is more conceptual, especially in the definition and the functorial properties of singular foliated homology (Section 3).

2.2. Standard embeddings. Let $\Sigma$ be an $\mathscr{R}$-simplicial complex. Pick a countable set $I$ and an isomorphism $\mathscr{R} \rightarrow X \times I$ of $X$-spaces [13].

Definition 2.11. Let $\boldsymbol{\Delta}(I)$ be the simplicial complex whose vertex set is $I$ and whose simplices consist of all finite subsets of $I$. The realization of $\boldsymbol{\Delta}(I)$ is denoted by the same symbol; to which meaning we refer should be clear from the context. 
Let $\mathscr{F}$ be a Borel fundamental domain of $\Sigma^{(0)}$. Then there is a countable Borel partition $\mathscr{F}=\coprod_{n \in \mathbb{N}} \mathscr{F}_{n}$ such that $p_{\Sigma}$ is injective on each $\mathscr{F}_{j}$ (theorem of selection; see e.g. [33, Lemma 3.1] for the version needed here). The injective map

$$
\Sigma^{(0)}=\mathscr{R} . \mathscr{F} \rightarrow \mathscr{R} \times \mathbb{N} \cong(X \times I) \times \mathbb{N}=X \times \Delta(I \times \mathbb{N})^{(0)},
$$

where the first arrow maps $(y, x) . u$ with $u \in \mathscr{F}_{n}$ and $x=p_{\Sigma}(u)$ to $(y, x, n)$ and the second arrow comes from the isomorphism above, extends to an embedding

$$
\Sigma \rightarrow X \times \Delta(I \times \mathbb{N})
$$

We say that (2.1) is a standard embedding of $\Sigma$. Note that under Assumption 1.1 we could take $I=\Gamma$.

2.3. $\mathscr{R}$-spaces and geometric $\mathscr{R}$-maps. The geometric realization $|\Sigma|$ of an $\mathscr{R}$ simplicial complex $\Sigma$ is the disjoint union of the geometric realizations $\left|\Sigma_{x}\right|$ of the fibers.

Definition 2.12. By definition, an $\mathscr{R}$-space is the geometric realization of some $\mathscr{R}$-simplicial complex $\Sigma$ such that $\Sigma_{x}$ is locally finite for a.e. $x \in X$. Here $\Sigma$ is part of the data of the $\mathscr{R}$-space.

A standard embedding $\phi$ as in (2.1) yields an injection $|\phi|:|\Sigma| \rightarrow X \times|\boldsymbol{\Delta}(I \times \mathbb{N})|$. The standard Borel structure of $|\Sigma|$ is defined as the restriction of the product Borel structure on $X \times|\boldsymbol{\Delta}(I \times \mathbb{N})|$.

Lemma 2.13. The Borel structure in the previous definition does not depend on the choice of a standard embedding, and $\operatorname{im}(|\phi|) \subset X \times|\boldsymbol{\Delta}(I \times \mathbb{N})|$ is a Borel subset.

We skip the proof of this easy lemma. Its first part follows from Lemma 2.14 below (whose proof is also left to the reader) and the fact that, if $I$ is countable, the subsets $A \times K$, where $A \subset X$ is Borel and $K \subset|\boldsymbol{\Delta}(I)|$ compact, form a basis of the Borel algebra of $X \times|\boldsymbol{\Delta}(I)|$.

\section{Lemma 2.14.}

a) Let $\phi: X \times I \rightarrow X \times J$ be an isomorphism of $X$-spaces. The induced bijection $|\phi|: X \times|\boldsymbol{\Delta}(I)| \rightarrow X \times|\boldsymbol{\Delta}(J)|$ has the following property: For every compact subset $K \subset|\boldsymbol{\Delta}(I)|$ there is a countable Borel partition of $X$ into sets $X_{n}$ such that $\mid \phi \|_{X_{n} \times K}$ is a product $\operatorname{id}_{X_{n}} \times f_{n}$. In particular, for every Borel subset $A \subset$ $X,|\phi|(A \times K)$ is a union of sets $A_{n} \times K_{n}$ with $K_{n}$ compact and $A_{n}=X_{n} \cap A$.

b) For an $\mathscr{R}$-simplicial complex $\Sigma$ let $\phi$ and $\theta$ be standard embeddings $\Sigma \rightarrow X \times$ $\boldsymbol{\Delta}(I \times \mathbb{N})$. Let $K \subset|\boldsymbol{\Delta}(I \times \mathbb{N})|$ be compact and $A \subset X$ be a Borel subset such that $A \times K \subset \operatorname{im}(|\phi|)$. Then there is a countable Borel partition of $A$ by sets $A_{n}$ such that each restriction $\left.|\theta| \circ|\phi|^{-1}\right|_{A_{n} \times K}$ is a product map $\operatorname{id}_{A_{n}} \times f_{n}$ with continuous $f_{n}: K \rightarrow|\boldsymbol{\Delta}(I \times \mathbb{N})|$.

Definition 2.15. The $n$-simplices of an $\mathscr{R}$-simplicial complex correspond to subspaces of its realization homeomorphic to $\Delta^{n}$, which we call geometric $n$-simplices.

Remark 2.16. We equip the fibers of an $\mathscr{R}$-space with the unique length metric that restricts to the standard Euclidean metric on geometric simplices. All metric notions refer to this metric. Since a.e. fiber is locally compact, the weak topology coincides with the topology obtained from the metric on a.e. fiber.

Remark 2.17. Note that we use Greek capital letters $(\Sigma, \Phi, \ldots)$ to denote both $\mathscr{R}$-spaces and $\mathscr{R}$-simplicial complexes. The $|\Sigma|$-notation is only used if we want to refer explicitly to the underlying $\mathscr{R}$-simplicial complex. 
Remark 2.18. We leave it to the reader to verify that the $\mathscr{R}$-action on $\Sigma$ induces an $\mathscr{R}$-action on $|\Sigma|$ in the same sense as in Definition 2.2 except map of $X$-spaces should be replaced by Borel map over $X$. This action is fiberwise continuous. The union of geometric simplices corresponding to fundamental domains of $\Sigma^{(n)}$ over all $n \geq 0$ is a Borel fundamental domain of $|\Sigma|$.

Definition 2.19. Since $\Sigma$ has an $\mathscr{R}$-fundamental domain, the restriction of the projection pr : $\Sigma_{x} \rightarrow \mathscr{R} \backslash \Sigma$ is injective for all $x \in X$. The image $\operatorname{pr}\left(\Sigma_{x}\right) \subset \mathscr{R} \backslash \Sigma$, which can be identified with $\Sigma_{x}$, is called the leaf at $x \in X$ and denoted by $\mathscr{L}_{\Sigma}(x)$.

The quotient $\mathscr{R} \backslash \Sigma$ can be seen as a foliated space, foliated by the leaves $\mathscr{L}_{\Sigma}(x)$.

Definition 2.20. Fix a choice of standard embeddings $\Sigma, \Phi \rightarrow X \times \Delta(I \times \mathbb{N})$. A map $\phi:|\Sigma| \rightarrow|\Phi|$ over $X$ is said to be of countable variance, if for any set $A \times K, A \subset X$ Borel, $K \subset|\boldsymbol{\Delta}(I \times \mathbb{N})|$ compact, there is a countable Borel partition $A=\bigcup_{n \in \mathbb{N}} A_{n}$ such that $A_{n} \times K \subset|\Sigma|$ and each restriction $\left.\phi\right|_{A_{n} \times K}$ is a product.

Independence of the chosen standard embeddings is implied by Lemma 2.14. Further, it is clear that countable variance implies measurability.

Definition 2.21. A geometric $\mathscr{R}$-map between $\mathscr{R}$-spaces is a map over $X$ of countable variance that is $\mathscr{R}$-equivariant (in the sense of Definition 2.7 ), continuous on a.e. fiber and proper on a.e. fiber. We say that a geometric $\mathscr{R}$-map between $\mathscr{R}$-spaces is $\mathscr{R}$-simplicial if it is the realization of an $\mathscr{R}$-simplicial map of the underlying $\mathscr{R}$ simplicial complexes.

Remark 2.22. A geometric $\mathscr{R}$-map $\phi: \Sigma \rightarrow \Phi$ induces proper maps $\mathscr{L}_{\Sigma}(x) \rightarrow$ $\mathscr{L}_{\Phi}(x)$ for a.e. $x \in X$.

It would be more natural and less cumbersome in some places to replace the condition of countable variance by measurability. However, for technical reasons we need countable variance at some places, notably in the proofs of Theorems 3.12 and 3.25 .

The easy proof (similar to Lemma 2.14) of the following lemma is left to the reader.

Lemma 2.23. Let $\Sigma, \Phi$ be $\mathscr{R}$-simplicial complexes with locally finite fibers a.e and $\phi: \Sigma \rightarrow \Phi$ an $\mathscr{R}$-simplicial map. If $\phi_{x}$ is proper for a.e. $x \in X$, then the realization $|\phi|$, defined fiberwise as $\left|\phi_{x}\right|:\left|\Sigma_{x}\right| \rightarrow\left|\Phi_{x}\right|$, is a geometric $\mathscr{R}$-map.

2.4. Simplicial approximation of geometric $\mathscr{R}$-maps. In this Subsection we introduce simplicial approximation theorems in the context of $\mathscr{R}$-space. We start by recalling some terminology concerning simplicial approximation. The smallest simplex in (the realization) of a simplicial complex that contains the point $m$ is denoted by $\operatorname{carr}(m)$. The open star of a vertex $v$ in a simplicial complex is denoted by $\operatorname{star}(v)$. Notice that $x \in \operatorname{star}(v) \Leftrightarrow v \in \operatorname{carr}(x)$. Let $f, g$ be two maps from a topological space $M$ to a simplicial complex $K$. Then $g$ is called an approximation of $f$ if $g(x) \in \operatorname{carr}(f(x))$ for all $x \in M$. If $M$ and $g$ are simplicial, then $g$ is called a simplicial approximation of $f$. If $\phi, \psi$ are geometric $\mathscr{R}$-maps between $\mathscr{R}$-spaces, we call $\phi$ an ( $\mathscr{R}$-simplicial) approximation of $\psi$ if $\phi_{x}$ is a (simplicial) approximation of $\psi_{x}$ for a.e. $x \in X$. The Lebesgue number of an open cover of a metric space is the supremum of all $r \geq 0$ such that every set of diameter less than $r$ is contained in an element of the cover.

Lemma 2.24. Let $\phi:|\Sigma| \rightarrow|\Phi|$ be a geometric $\mathscr{R}$-map. Let $\psi:|\Sigma| \rightarrow|\Phi|$ be an $\mathscr{R}$-equivariant map that is of countable variance and continuous in a.e. fiber. Suppose that $\psi_{x}$ is an approximation of $\phi_{x}$ for a.e. $x \in X$. Then $\psi_{x}$ is proper for a.e. $x \in X$, thus $\psi$ is a geometric $\mathscr{R}$-map. Furthermore, there is a geometric $\mathscr{R}$-homotopy between $\phi$ and $\psi$. 
Proof. Define $H:|\Sigma| \times[0,1] \rightarrow|\Phi|$ to be the map such that $\left.H\right|_{\{m\} \times[0,1]}$ parametrizes the straight line segment connecting $\psi(m)$ and $\phi(m)$ (within the simplex $\operatorname{carr}(\phi(m))$ ). Equivariance of $H$ is clear. First we verify that $H_{x}$ is proper (in particular, $\psi_{x}$ is). Let $K \subset\left|\Phi_{x}\right|$ be a compact subcomplex and $\left\{k_{1}, \ldots, k_{p}\right\}$ be the finitely many vertices of $K$. We show that

$$
H_{x}^{-1}(K) \subset \bigcup_{i=1}^{p} \phi_{x}^{-1}\left(\operatorname{star}\left(k_{i}\right)\right) \times[0,1] .
$$

Let $(m, t) \in H_{x}^{-1}(K)$. Then

$$
H_{x}(m, t) \in \operatorname{carr}\left(\phi_{x}(m)\right) \cap K \neq \emptyset .
$$

There is a vertex $k_{i}$ in $K$ such that $k_{i} \in \operatorname{carr}\left(\phi_{x}(m)\right)$, thus $m \in \phi_{x}^{-1}\left(\operatorname{star}\left(k_{i}\right)\right)$ showing (2.2). Since $\Phi_{x}$ is locally finite and $\phi_{x}$ is proper, $(2.2)$ implies that $H_{x}^{-1}(K)$ is compact. Next we verify that $H$ is of countable variance. Choose standard embeddings $\Sigma, \Phi \rightarrow X \times \Delta(I \times \mathbb{N})$. Let $K \subset \boldsymbol{\Delta}(I \times \mathbb{N})$ be a compact subcomplex. Let $\left\{A_{n}\right\}_{n \in \mathbb{N}}$ and $\left\{B_{n}\right\}_{n \in \mathbb{N}}$ be Borel partitions of $X$ such that $\left.\phi\right|_{A_{n} \times K}$ and $\left.\psi\right|_{B_{n} \times K}$ are product maps. If $\left\{C_{n}\right\}_{n \in \mathbb{N}}$ is a refinement of $\left\{A_{n}\right\}_{n \in \mathbb{N}}$ and $\left\{B_{n}\right\}_{n \in \mathbb{N}}$, then $\left.H\right|_{C_{n} \times(K \times[0,1])}$ is a product map for all $n \in \mathbb{N}$. So $H$ is the geometric $\mathscr{R}$-homotopy between $\phi$ and $\psi$.

Definition 2.25. Let $\phi:|\Sigma| \rightarrow|\Phi|$ be a geometric $\mathscr{R}$-map. Let $L(x)$ be the Lebesgue number of the pullback under $\phi_{x}$ of the open star cover of $\left|\Phi_{x}\right|$. The Lebesgue number of $\phi$ is defined as the essential infimum of $\{L(x) ; x \in X\}$.

Theorem 2.26. Let $\phi:|\Sigma| \rightarrow|\Phi|$ be a geometric $\mathscr{R}$-map. If the Lebesgue number of $\phi$ is positive, then there is an $n \in \mathbb{N}$ and an $\mathscr{R}$-simplicial approximation $\psi: \operatorname{sd}^{(n)}(\Sigma) \rightarrow \Phi$ of $\phi$. Further, $|\psi|$ is a geometric $\mathscr{R}$-map, and $\phi$ and $|\psi|$ are geometrically $\mathscr{R}$-homotopic.

Proof. We examine the classical proof to see that the simplicial approximations on the fibers assemble to an $\mathscr{R}$-simplicial map.

Let $\delta>0$ be the Lebesgue number of $\phi$. Take $n \in \mathbb{N}$ large enough so that the diameter of a (geometric) simplex in the $n$-fold barycentric subdivision of $\left|\Sigma_{x}\right|$ with respect to the length metric of $\left|\Sigma_{x}\right|$ is less than $\delta / 2$ for a.e. $x \in X$. Then the diameter of the open star of a vertex $v$ in $\left|\operatorname{sd}^{(n)}(\Sigma)_{x}\right|=\left|\Sigma_{x}\right|$, is less than $\delta$, thus its image under $\phi$ is contained in the open star of some vertex $w$ of $\left|\Phi_{x}\right|$. For purposes of the proof we can now forget $n$ and assume that the image of an open star in $\left|\Sigma_{x}\right|$ is contained in some open star of $\left|\Phi_{x}\right|$.

Pick standard embeddings $\Sigma, \Phi \rightarrow X \times \Delta(I \times \mathbb{N})$. Let $\mathscr{F}$ be a Borel fundamental domain for $\Sigma^{(0)} \subset X \times(I \times \mathbb{N}) ; \mathscr{F}$ is the disjoint union of sets $A_{k} \times\left\{\left(i_{k}, n_{k}\right)\right\}$, $k \in \mathbb{N}$, with $A_{k} \subset X$ Borel, $i_{k} \in I$ and $n_{k} \in \mathbb{N}$. For a.e. $x \in X$ and every $k \in \mathbb{N}$ there are $j \in I$ and $m \in \mathbb{N}$ such that we have for the open stars

$$
\phi_{x}\left(\operatorname{star}\left(x, i_{k}, n_{k}\right)\right) \subset \operatorname{star}(x, j, m) .
$$

Since for fixed $k \in \mathbb{N}$ and $x$ running through $A_{k}$ there are only countable many $(j, m)$ appearing in (2.3), we can assume after further refining the Borel partition $\left(A_{k}\right)_{k \in \mathbb{N}}$ that for every $k \in \mathbb{N}$ there are $j=j_{k} \in I$ and $m=m_{k} \in \mathbb{N}$ such that (2.3) holds for a.e. $x \in A_{k}$.

Define $\psi^{(0)}$ on $\mathscr{F}$ by sending $\left(x, i_{k}, n_{k}\right)$ to $\left(x, j_{k}, m_{k}\right)$ for $x \in A_{k}$, and extend it to $\Sigma^{(0)}$ by equivariance. It follows (fiberwise) from the classical proof [37, Theorem 3 on p. 127] that the map induced by $\psi$ between the $n$-fold fibered products of $\Sigma^{(0)}, \Phi^{(0)}$ restricts to $\Sigma^{(n)} \rightarrow \Phi^{(n)}$ for any $n \geq 0$. The rest follows from Lemma 2.24 . 
2.5. $\mathscr{R}$-covers. Throughout Subsection 2.5, we retain the notation of Assumption 1.2. We introduce certain equivariant covers, so-called $\mathscr{R}$-covers, on the $\mathscr{R}$ space $X \times \widetilde{M}$ and a nerve construction.

Definition 2.27. Let $I$ be a free $\Gamma$-set. For $i \in I$ let $A_{i} \subset X$ be a Borel subset and and $U_{i} \subset \widetilde{M}$ be an open subset.

The family $\mathscr{U}=\left\{A_{i} \times U_{i}\right\}_{i \in I}$ is called an $\mathscr{R}$-cover of $X \times \widetilde{M}$ if

a) $A_{\gamma i}=\gamma A_{i}, U_{\gamma i}=\gamma U_{i}$ for all $\gamma \in \Gamma$ and $i \in I$,

b) $\mathscr{U}_{x}:=\left\{U_{i} ; x \in A_{i}\right\}_{i \in I}$ is locally finite in $\widetilde{M}$ for a.e. $x \in X$,

c) for fixed $m \in \widetilde{M}$ and for a.e. $x \in X$ it is $(x, m) \in \bigcup_{i \in I} A_{i} \times U_{i} \subset X \times \widetilde{M}$.

The family $\mathscr{U}=\left\{A_{i} \times U_{i}\right\}_{i \in I}$ is called an $\mathscr{R}$-packing if

a') $A_{\gamma i}=\gamma A_{i}, U_{\gamma i}=\gamma U_{i}$ for all $\gamma \in \Gamma$ and $i \in I$,

b') $U_{i} \cap U_{j} \neq \emptyset \Rightarrow \mu\left(A_{i} \cap A_{j}\right)=0$ for all $i \neq j$ in $I$.

Lemma 2.28. Every $\mathscr{R}$-cover $\mathscr{U}=\left\{A_{i} \times U_{i}\right\}_{i \in I}$ of $X \times \widetilde{M}$ such that $\mu\left(A_{i}\right)>0$ for every $i \in I$ has the following properties.

a) The index set I is countable.

b) For every compact $K \subset \widetilde{M}$ there is a countable Borel partition $X=\bigcup_{j \in J} X_{j}$ such that for almost all $x, y \in X_{j}$ and every $k \in K$ we have: $(x, k) \in A_{i} \times U_{i} \Leftrightarrow$ $(y, k) \in A_{i} \times U_{i}$

c) $\mathscr{U}_{x}$ is a cover of $\widetilde{M}=\{x\} \times \widetilde{M}$ for a.e. $x \in X$.

Proof. Let $T \subset \widetilde{M}$ be a countable dense subset, and set $I_{m}=\left\{i \in I ; m \in U_{i}\right\}$ for $m \in T$. Then $I=\bigcup_{m \in T} I_{m}$. For any $m \in T$ we define a Borel partition $X=\bigcup_{n \geq 1} X_{m}(n)$ by

$$
X_{m}(n)=\left\{x \in X ; \#\left\{i \in I ;(x, m) \in A_{i} \times U_{i}\right\}=n\right\} .
$$

Note here that since $\mathscr{U}_{x}$ is locally finite, the sets $\left\{i \in I ;(x, m) \in A_{i} \times U_{i}\right\}$ are finite for a.e. $x \in X$. Each set $I_{m}(r, s)=\left\{i \in I_{m} ; \mu\left(X_{m}(r) \cap A_{i}\right)>\frac{1}{s}\right\}$ is finite since $\sum_{i \in I_{m}(r, s)} \mu\left(X_{m}(r) \cap A_{i}\right) \leq r \mu\left(X_{m}(r)\right)<\infty$. That $I$ is countable follows from

$$
I=\bigcup_{m \in T} \bigcup_{r, s \geq 1} I_{m}(r, s)
$$

Set $I_{K}(x)=\left\{i \in I ; x \in A_{i}, K \cap U_{i} \neq \emptyset\right\}$. Since $\mathscr{U}_{x}$ is locally finite $I_{K}(x)$ is finite for a.e. $x \in X$. In particular, $I_{K}$ ranges as a function of $x$ in the countable set of finite subsets of $I$. Let $X=\bigcup_{j \in J} X_{j}$ be a countable Borel partition such that $I_{K}(x)$ is a constant set on each $X_{j}$. This proves the second assertion.

To prove the third assertion, consider a compact subset $K \subset \widetilde{M}$ such that $\Gamma K=\widetilde{M}$. Let $X=\bigcup_{j \in J} X_{j}$ of $X$ be as in the second assertion. Suppose $\mathscr{U}_{x}$ is not a cover, i.e. $\{x\} \times \widetilde{M} \not \subset \bigcup_{i \in I} A_{i} \times U_{i}$ on a subset of positive measure. This implies that $\{x\} \times K \not \subset \bigcup_{i \in I} A_{i} \times U_{i}$ on a subset $Y \subset X$ of positive measure that has a non-trivial intersection with some $X_{j_{0}}$. Pick $y_{0} \in Y \cap X_{j_{0}}$. Let $m \in K$ be such that $\left(y_{0}, m\right) \notin \bigcup_{i \in I} A_{i} \times U_{i}$. Then $(y, m) \notin \bigcup_{i \in I} A_{i} \times U_{i}$ for a.e. $y \in Y \cap X_{j_{0}}$ contradicting c) in Definition 2.27.

Lemma 2.29. Let $\mathscr{U}=\left\{A_{i} \times U_{i}\right\}_{i \in I}$ be an $\mathscr{R}$-packing such that $\mu\left(A_{i}\right)>0$ for every $i \in I$. Then

a) $I$ is countable, and

b) for a.e. $x \in X$ the sets in $\left\{U_{i} ; x \in A_{i}\right\}$ are pairwise disjoint. That is, $\mathscr{U}_{x}$ is a packing for a.e. $x \in X$. 
Proof. Let $T$ and $I_{m}$ for $m \in T$ be like in the previous proof. Then $I(m)$ has to be countable since otherwise there would exist $i \neq j$ in $I$ with $\mu\left(A_{i} \cap A_{j}\right)>0$. Thus, $I$ is countable.

Suppose there is a Borel subset $A \subset X$ with $\mu(A)>0$ such that for every $x \in A$ there are $i \neq j$ with $x \in A_{i} \cap A_{j}$ and $U_{i} \cap U_{j} \neq \emptyset$. We may assume that $I=\mathbb{N}$. For $x \in A$, let $i(x)<j(x)$ be minimal in $I$ with this property. Since $I \times I$ is countable, there is a Borel subset $B \subset A$ with $\mu(B)>0$ such that $(i(x), j(x))$ is constant for $x \in B$, which contradicts c') in Definition 2.27.

Definition 2.30. The nerve $\mathscr{N}(\mathscr{U})$ of an $\mathscr{R}$-cover $\mathscr{U}=\left\{A_{i} \times U_{i}\right\}_{i \in I}$ of $X \times \widetilde{M}$ is the $\mathscr{R}$-simplicial complex whose 0 -simplices are

$$
\mathscr{N}(\mathscr{U})^{(0)}=\left\{(x, i) ; x \in A_{i}, i \in I\right\} \subset X \times I .
$$

and whose set of $n$-simplices $\mathscr{N}(\mathscr{U})^{(n)} \subset \mathscr{N}(\mathscr{U})^{(0)} * \ldots * \mathscr{N}(\mathscr{U})^{(0)}$ is given by

$$
\mathscr{N}(\mathscr{U})^{(n)}=\left\{\left(x, i_{0}, \ldots, i_{n}\right) ; x \in \bigcap_{s=0}^{n} A_{i_{s}}, \bigcap_{s=0}^{n} U_{i_{s}} \neq \emptyset, i_{k} \neq i_{l} \text { for } k \neq l\right\} .
$$

The nerve $\mathscr{N}(\mathscr{U})$ carries the $\mathscr{R}$-action that comes from the $\Gamma$-action $\gamma\left(x, i_{0}, \ldots, i_{n}\right)=$ $\left(\gamma x, \gamma i_{0}, \ldots, \gamma i_{n}\right)$ (cf. Remark 2.3).

Remark 2.31. The map $\mathscr{N}(\mathscr{U}) \rightarrow X \times \Delta(I),\left(x, i_{0}, \ldots, i_{n}\right) \mapsto\left(x, i_{0}, \ldots, i_{n}\right)$, is a standard embedding in the sense of Section 2.2 .

Remark 2.32. By Lemma 2.28, $\mathscr{U}_{x}$ is a cover of $\widetilde{M}$ for a.e. $x \in X$, and $\mathscr{N}(\mathscr{U})_{x}$ is the nerve of the cover $\mathscr{U}_{x}$, which is locally finite for a.e. $x \in X$ since $\mathscr{U}_{x}$ is so.

Remark 2.33. The $\mathscr{R}$-space obtained from the realization of $\mathscr{N}(\mathscr{U})$ is also called the nerve of $\mathscr{U}$ and denoted by the same notation. It will be clear from the context to which meaning we refer.

Lemma 2.34. Let $\mathscr{U}=\left\{A_{i} \times U_{i}\right\}_{i \in I}$ be an $\mathscr{R}$-cover. Let $I^{\prime} \subset I$ be a complete set of $\Gamma$-representatives. Set

$$
f\left(i_{0}, \ldots, i_{n}\right)= \begin{cases}\mu\left(A_{i_{0}} \cap \ldots \cap A_{i_{n}}\right) & \text { if } i_{l} \neq i_{k} \text { for } l \neq k \text { and } \bigcap_{k=0}^{n} U_{i_{k}} \neq \emptyset, \\ 0 & \text { otherwise. }\end{cases}
$$

Then we have (cf. Definition 2.9)

$$
\sigma_{n}(\mathscr{N}(\mathscr{U}))=\frac{1}{(n+1) !} \cdot \nu_{t}\left(\mathscr{R} \backslash \mathscr{N}(\mathscr{U})^{(n)}\right)=\sum_{i_{0} \in I^{\prime}} \sum_{\left(i_{1}, \ldots, i_{n}\right) \in I^{n}} f\left(i_{0}, \ldots, i_{n}\right) .
$$

Proof. It is clear that

$$
\mathscr{F}=\left\{\left(x, i_{0}, i_{1}, \ldots, i_{n}\right) \in \mathscr{N}(\mathscr{U})^{(n)} ; i_{0} \in I^{\prime}\right\}
$$

is an $\mathscr{R}$-fundamental domain of $\mathscr{N}(\mathscr{U})^{(n)}$. Let $\mathscr{F}_{x}=\mathscr{F} \cap \mathscr{N}(\mathscr{U})_{x}$. From Definition 2.5 we see that

$$
\nu_{t}\left(\mathscr{R} \backslash \mathscr{N}(\mathscr{U})^{(n)}\right)=\int_{X} \# \mathscr{F}_{x} d \mu(x) .
$$

The right hand side in (2.6) coincides with (2.4) by Fubini's theorem applied to the product measure space $X \times I^{(n+1)}$ with the counting measure on $I^{(n+1)}$.

For the following, recall that all metric notions on simplicial complexes refer to the length metric that restricts to the standard Euclidean metric on simplices.

Lemma 2.35. Let $\mathscr{U}=\left\{A_{i} \times U_{i}\right\}_{i \in I}$ be an $\mathscr{R}$-cover of $X \times \widetilde{M}$ such that $\Gamma \backslash I$ is finite. Then there is a geometric $\mathscr{R}$-map $\phi: X \times \widetilde{M} \rightarrow \mathscr{N}(\mathscr{U})$ such that a) $\phi$ is $\mathscr{R}$-simplicial (after a multiple barycentric subdivision of the domain). 
b) For a.e. $x \in X$ the preimage under $\phi_{x}$ of the open star of the vertex $i \in I$ in $\mathscr{N}(\mathscr{U})_{x}$ is contained in $U_{i}$.

Proof. Let $K \subset \widetilde{M}$ be a compact set that contains the open 1-neighborhood of a $\Gamma$-fundamental domain of $\widetilde{M}$. Then the cover $\{\gamma K ; \gamma \in \Gamma\}$ of $\widetilde{M}$ has Lebesgue number 1 . Since $\Gamma \backslash I$ is finite and $\Gamma$ acts properly on $\widetilde{M}$, there can be only finitely many $i \in I$ with $K \cap U_{i} \neq \emptyset$. In particular, there is a subset $X^{\prime} \subset X$ of full measure such that only finitely many covers appear as the restriction of some $\mathscr{U}_{x}$, $x \in X^{\prime}$, to $K$. Each of them has positive Lebesgue number with respect to restricted metric on $K$. If $\epsilon^{\prime}>0$ is the minimal such number, then $\mathscr{U}_{x}$ has Lebesgue number $\epsilon:=\min \left\{\epsilon^{\prime}, 1\right\}$ for a.e. $x \in X$.

For each $U_{i}$, let $\bar{B}_{\epsilon / 4}\left(\partial U_{i}\right)$ be the closed $\epsilon / 4$-neighborhood of the boundary $\partial U_{i}$. Set $V_{i}:=U_{i}-\bar{B}_{\epsilon / 4}\left(\partial U_{i}\right)$. Then $\mathscr{V}=\left\{A_{i} \times V_{i}\right\}_{i \in I}$ is an $\mathscr{R}$-cover of $X \times \widetilde{M}$ such that $\mathscr{V}_{x}$ has Lebesgue number $\epsilon / 4$ and $\bar{V}_{i} \subset U_{i}$. Let $I^{\prime} \subset I$ be a system of $\Gamma$ representatives. By Uryson's lemma, for each $i \in I^{\prime}$ there is a function $\tau_{i}: \widetilde{M} \rightarrow$ $[0,1]$ such that $\left.\tau_{i}\right|_{\bar{V}_{i}} \equiv 1$ and $\operatorname{supp}\left(\tau_{i}\right) \subset U_{i}$. Extend the definition of $\tau_{i}$ to all $i \in I$ by $\tau_{\gamma i}(m)=\tau_{i}\left(\gamma^{-1} m\right)$. Now define $\tau: X \times \widetilde{M} \rightarrow \mathscr{N}(\mathscr{U})$ by

$$
\tau(x, m)=\left(x, \frac{1}{\sum_{i \in I} \chi_{A_{i}}(x) \tau_{i}(m)} \sum_{i \in I} \chi_{A_{i}}(x) \tau_{i}(m) i\right) .
$$

Here $\chi_{A_{i}}$ denotes the characteristic function of $A_{i}$. Then $\tau$ is proper as $\tau$ clearly satisfies the second assertion and has Lebesgue number $\epsilon / 4$. Obviously, $\tau$ is equivariant. Countable variance follows from Lemma $2.28 \mathrm{~b}$ ).

By Theorem 2.26, $\tau$ possesses a simplicial approximation $\phi$, which still satisfies the second assertion.

Definition 2.36. An $\mathscr{R}$-cover $\mathscr{U}=\left\{A_{i} \times U_{i}\right\}_{i \in I}$ of $X \times \widetilde{M}$ is called uniformly bounded if there is an $R>0$ such that $\operatorname{diam}\left(U_{i}\right)<R$ for every $i \in I$.

Definition 2.37. A geometric $\mathscr{R}$-map $\phi: \Sigma \rightarrow \Phi$ between $\mathscr{R}$-spaces $\Sigma$ and $\Phi$ is called metrically coarse if for all $R>0$ there is an $S>0$ such that for a.e. $x \in X$ and for all $m, m^{\prime} \in \Sigma_{x}$ we have

$$
d\left(m, m^{\prime}\right)<R \Rightarrow d\left(\phi(m), \phi\left(m^{\prime}\right)\right)<S .
$$

Theorem 2.38. Assume that $M$ is aspherical. Let $\Sigma$ be a finite-dimensional $\mathscr{R}$ simplicial complex and $\Phi \subset \Sigma$ an $\mathscr{R}$-simplicial subcomplex that contains $\Sigma^{(0)}$. Let $\phi:|\Phi| \rightarrow X \times \widetilde{M}$ be a metrically coarse geometric $\mathscr{R}$-map. Then $\phi$ can be extended to a metrically coarse geometric $\mathscr{R}$-map $\phi:|\Sigma| \rightarrow X \times \widetilde{M}$.

Proof. Let $n \geq 1$. We show how to extend $\phi$ from $\left|\Sigma^{(n-1)}\right| \cup|\Phi|$ to $\left|\Sigma^{n}\right| \cup|\Phi| \subset|\Sigma|$. Let $\mathscr{F}$ be a fundamental domain for the $\mathscr{R} \times \mathbf{S}(n+1)$-action on $\Sigma^{(n)} \backslash \Phi^{(n)}$. There is a countable partition $\mathscr{F}=\coprod_{j \in J} \mathscr{F}_{j}$ such that the projection $\mathscr{F} \rightarrow X$ is injective on each $\mathscr{F}_{j}\left[33\right.$, Lemma 3.1]. Let $X_{j} \subset X$ be the image of $\mathscr{F}_{j}$. For every $j \in J$ there is an embedding $X_{j} \times e_{j} \rightarrow\left|\Sigma^{(n)}\right|$ with $e_{j}=\left|\Delta^{n}\right|$ whose restriction to $\{x\} \times e_{j}$ is an affine isomorphism onto the (geometric) simplex given by the unique element in $\mathscr{F}_{j} \cap \Phi_{x}$ for $x \in X_{j}$. Identifying $X_{j} \times e_{j}$ with its image, we can write:

$$
\left|\Sigma^{(n)}\right| \cup|\Phi|=\bigcup_{j \in J} \mathscr{R} .\left(X_{i} \times e_{j}\right) \cup\left|\Sigma^{(n-1)}\right| \cup|\Phi|
$$

Since $\phi$ (as defined so far) is of countable variance, we can assume, after possibly refining $\left(X_{j}\right)_{i \in J}$, that $\left.\phi\right|_{X_{j} \times \partial e_{j}}$ is a product id $\times f_{j}$ with continuous $f_{j}: \partial e_{j} \rightarrow \widetilde{M}$. Since $\phi$ is metrically coarse, the diameters of $f_{j}\left(\partial e_{j}\right)$ are uniformly bounded by a constant $R$. Note that $\widetilde{M}$ is uniformly contractible because $M$ is compact and 
aspherical. That is, every $R$-ball of $\widetilde{M}$ can be contracted within an $S$-ball for some $S>0$. So we can find an extension $F_{j}: e_{j} \rightarrow \widetilde{M}$ of $f_{j}$ for every $j \in J$ such that $F_{j}\left(e_{j}\right)$ has diameter at most $S$, thus obtaining a metrically coarse extension of $\phi$ to $\left|\Sigma^{(n)}\right| \cup|\Phi|$ by equivariance. Countable variance of that extension is easy to verify.

It remains to show that $\phi:\left|\Sigma^{(n)}\right| \cup|\Phi| \rightarrow X \times \widetilde{M}$ is fiberwise proper. This follows from the following general statement [2, Lemma 4.1; 24, Lemma 3.3]:

Let $f: M \rightarrow N$ be a metrically coarse map between finite-dimensional, locally finite simplicial complexes $M$ and $N$ such that $\left.f\right|_{M^{(0)}}$ is proper, then $f$ is proper. Since every point of $M$ has distance at most 1 from $M^{(0)}$ and $M$ is locally finite, a subset $K \subset M$ is relatively compact if and only if $B_{1}(K) \cap M^{(0)}$ is finite. Here $B_{1}(K)$ denotes the 1-neighborhood of $K$. Now let $B(n, r) \subset N$ be the ball of radius $r$ around some $n \in N$. Because $f$ is metrically coarse, there is an $S>0$ such that $B_{1}\left(f^{-1}(B(n, r))\right)$ is contained in $f^{-1}(B(n, r+S))$. Since $f$ is proper on the 0 -skeleton,

$$
B_{1}\left(f^{-1}(B(n, r))\right) \cap M^{(0)} \subset f^{-1}(B(n, r+S)) \cap M^{(0)}
$$

is finite. Thus $f^{-1}(B(n, r))$ is relatively compact, and $f$ is proper.

Lemma 2.39. Let $M$ be aspherical. Let $\mathscr{U}=\left\{A_{i} \times U_{i}\right\}_{i \in I}$ be a uniformly bounded $\mathscr{R}$-cover of $X \times \widetilde{M}$. Suppose $\mathscr{N}(\mathscr{U})_{x}$ is finite-dimensional for a.e. $x \in X$. Then there is a metrically coarse, geometric $\mathscr{R}$-map $\psi: \mathscr{N}(\mathscr{U}) \rightarrow X \times \widetilde{M}$.

Proof. Let $I^{\prime} \subset I$ a set of $\Gamma$-representatives. Pick for every $i \in I^{\prime}$ a point $m_{i} \in U_{i}$, and extend the definition of $m_{i}$ to $i \in I$ by $\gamma m_{i}=m_{\gamma i}$. Define $\psi: \mathscr{N}(\mathscr{U})^{(0)} \rightarrow$ $X \times \widetilde{M}$ by sending $(x, i)$ with $x \in A_{i}$ and $i \in I$ to $\left(x, m_{i}\right)$. For a.e. $x \in X$ the map $\psi_{x}$ is proper since $\mathscr{U}_{x}$ is locally finite, and $\psi$ is metrically coarse since $\mathscr{U}$ is uniformly bounded. Now extend $\psi$ to $\mathscr{N}(\mathscr{U})$ using Theorem 2.38 .

Lemma 2.40. Assume that $M$ is aspherical. Let $\phi: X \times \widetilde{M} \rightarrow X \times \widetilde{M}$ be a metrically coarse, geometric $\mathscr{R}$-map. Then there is a geometric $\mathscr{R}$-homotopy between $\operatorname{id}_{X \times \widetilde{M}}$ and $\phi$.

Proof. Apply Theorem 2.38 to extend the map $\phi \sqcup \mathrm{id}: X \times \widetilde{M} \times\{0,1\} \rightarrow X \times \widetilde{M}$ to $X \times \widetilde{M} \times[0,1]$.

\section{Singular foliated homology}

3.1. The bundle of singular simplices of an $\mathscr{R}$-space. The bundle of singular $n$-simplices of an $\mathscr{R}$-space $\Sigma$ is the disjoint union

$$
\operatorname{Sing}_{n}(\Sigma)=\coprod_{x \in X} \operatorname{Sing}_{n}\left(\Sigma_{x}\right)
$$

where $\operatorname{Sing}_{n}\left(\Sigma_{x}\right)$ is the set of singular $n$-simplices of $\Sigma_{x}$. The $\mathscr{R}$-action on $\Sigma$ induces one on $\operatorname{Sing}_{n}(\Sigma)$. Let pr : $\operatorname{Sing}_{n}(\Sigma) \rightarrow X$ denote the natural projection. Since the $\mathscr{R}$ space $\Sigma$ has an $\mathscr{R}$-fundamental domain (see Remark 2.18), $\operatorname{Sing}_{n}(\Sigma)$ has one as well: Take e.g. the set of singular $n$-simplices whose first vertex lies in the fundamental domain of $\Sigma$.

Note that in the case $\Sigma=X \times \widetilde{M}$ we have $\operatorname{Sing}_{n}(\Sigma)=X \times \operatorname{Sing}_{n}(\widetilde{M})$.

Remark 3.1. Let $\Phi$ be an $\mathscr{R}$-simplicial complex. Then there is a natural inclusion $\Phi^{(n)} \subset \operatorname{Sing}_{n}(|\Phi|)$ that maps $\left(v_{0}, \ldots, v_{n}\right) \in \Phi_{x}^{(n)} \subset \Phi_{x}^{(0)} * \ldots * \Phi_{x}^{(n)}$ to the singular $n$-simplex $\Delta^{n} \rightarrow\left|\Phi_{x}\right|,\left(t_{0}, \ldots, t_{n+1}\right) \mapsto t_{0} v_{0}+\ldots+t_{n} v_{n}$.

Of course, $\operatorname{Sing}_{n}(\Sigma)$ for an $\mathscr{R}$-space $\Sigma$ is not an $X$-space in the sense of Definition since its fibers are in general uncountable. Although Definition 2.5 thus cannot be 
applied, we will define a transversal measure for certain subsets of $\mathscr{R} \backslash \operatorname{Sing}_{n}(\Sigma)$ : From a standard embedding we obtain an injection

$$
\Phi: \operatorname{Sing}_{n}(\Sigma) \rightarrow X \times \operatorname{Sing}_{n}(\Delta(I \times \mathbb{N}))
$$

with respect to which we define the following notion.

Definition 3.2. A subset $W \subset \operatorname{Sing}_{n}(\Sigma)$ is admissible, if there exists a countable subset $C \subset \operatorname{Sing}_{n}(\boldsymbol{\Delta}(I \times \mathbb{N}))$ such that $\Phi(W)$ is a Borel subset of the $X$-space $X \times C$. A subset $W \subset \mathscr{R} \backslash \operatorname{Sing}_{n}(\Sigma)$ is admissible if its pullback to $\operatorname{Sing}_{n}(\Sigma)$ is admissible.

By Lemma 3.5 below the property of being admissible and the Borel structure of an admissible set do not depend on the choice of (3.1).

Remark 3.3. Obviously, intersections and countable unions of admissible sets are admissible. An admissible subset of $\operatorname{Sing}_{n}(\Sigma)$ is an $X$-space with respect to pr : $\operatorname{Sing}_{n}(\Sigma) \rightarrow X$ and carries, provided it is $\mathscr{R}$-invariant, a discrete $\mathscr{R}$-action since $\operatorname{Sing}_{n}(\Sigma)$ possesses a fundamental domain.

Definition 3.4. Let $\Sigma$ be an $\mathscr{R}$-space. For admissible $W \subset \operatorname{Sing}_{n}(\Sigma)$ the function $x \mapsto \#\left(\operatorname{pr}^{-1}(x) \cap W\right)$ on $X$ is integrable, and

$$
\nu(W)=\int_{X} \#\left(\operatorname{pr}^{-1}(x) \cap W\right) d \mu(x)
$$

is called the natural measure of $W$. Of course, $\nu(W)$ equals the natural measure of $\Phi(W) \subset X \times C$ defined in Definition 2.4. As in Definition 2.5 one defines the transversal measure $\nu_{t}(W)$ of an admissible subset $W \subset \mathscr{R} \backslash \operatorname{Sing}_{n}(\Sigma)$.

\section{Lemma 3.5.}

a) Consider embeddings $\Phi_{i}: \operatorname{Sing}_{n}(\Sigma) \rightarrow X \times \operatorname{Sing}_{n}\left(\Delta\left(I_{i} \times \mathbb{N}\right)\right), i \in\{1,2\}$, as in (3.1). Then $\Phi_{1} \circ \Phi_{2}^{-1}: \operatorname{Sing}_{n}(\Sigma) \rightarrow \operatorname{Sing}_{n}(\Sigma)$ maps admissible to admissible sets, and is Borel on admissible sets.

b) Let $\phi: \Sigma \rightarrow \Phi$ be a geometric $\mathscr{R}$-map. The map $\operatorname{Sing}_{n}(\phi): \operatorname{Sing}_{n}(\Sigma) \rightarrow \operatorname{Sing}_{n}(\Phi)$ induced by $\phi$ maps admissible to admissible sets, and is Borel on admissible sets.

Proof. We leave it to the reader to verify the assertions using Lemma 2.14 and the fact that the image of a singular simplex is compact.

3.2. Singular homology of $\mathscr{R}$-spaces. The goal of this section is to introduce the singular foliated homology $\mathscr{H}_{n}(\Sigma)$ of an $\mathscr{R}$-space $\Sigma$. In spite of the notation one should think of $\mathscr{H}_{n}(\Sigma)$ as being a homology group of the foliated space $\mathscr{R} \backslash \Sigma$. In fact, $\mathscr{H}_{n}(\Sigma)$ will be a singular version of the sheaf-theoretic tangential homology of measured foliations [29]. A homology class in $\mathscr{H}_{n}(\Sigma)$ gives rise to a measurable family of classes in the locally finite homology of the leaves $\mathscr{L}_{\Sigma}(x)$ (see Definition 2.19). However, the functorial properties (and the actual definition) of $\mathscr{H}_{*}$ can be easier given for $\mathscr{R}$-spaces than for their quotients.

In the following $\Sigma$ always denotes an $\mathscr{R}$-space. The restriction of the projection $\operatorname{Sing}_{n}(\Sigma) \rightarrow \mathscr{R} \backslash \operatorname{Sing}_{n}(\Sigma)$ to $\operatorname{Sing}_{n}\left(\Sigma_{x}\right)$ is injective for $x \in X$. By the identification $\mathscr{L}_{\Sigma}(x) \cong \Sigma_{x}$ we obtain an injection $\operatorname{Sing}_{n}\left(\mathscr{L}_{\Sigma}(x)\right) \rightarrow \mathscr{R} \backslash \operatorname{Sing}_{n}(\Sigma)$ that only depends on the $\mathscr{R}$-class of $x \in X$, that is, only on $\mathscr{L}_{\Sigma}(x)$ itself. Identifying $\operatorname{Sing}_{n}\left(\mathscr{L}_{\Sigma}(x)\right)$ with its image, we can view $\operatorname{Sing}_{n}\left(\mathscr{L}_{\Sigma}(x)\right)$ as a subset of $\mathscr{R} \backslash \operatorname{Sing}_{n}(\Sigma)$.

Notice that, if the $\mathscr{R}$-action comes from a $\Gamma$-action (cf. Remark 2.3), then $\mathscr{R} \backslash \operatorname{Sing}_{n}(\Sigma)=\Gamma \backslash \operatorname{Sing}_{n}(\Sigma)$ and $\mathscr{R} \backslash \operatorname{Sing}_{n}(\Sigma)=\Gamma \backslash \operatorname{Sing}_{n}(\Sigma)$.

Definition 3.6. A map $\sigma: A \rightarrow \mathscr{R} \backslash \operatorname{Sing}_{n}(\Sigma)$, where $A \subset \mathbb{R}$ is a Borel subset of finite Lebesgue measure, is called a foliated singular $n$-simplex of $\Sigma$, if it has the following properties: 
a) The image $\operatorname{im}(\sigma)$ is admissible, and $\sigma: A \rightarrow \operatorname{im}(\sigma)$ is an $\left(\operatorname{im}(\sigma), \nu_{t}\right)$-space in the sense of Definition 2.1.

b) The Lebesgue measure coincides with the natural measure of the $\left(\operatorname{im}(\sigma), \nu_{t}\right)$ space $A$.

c) The set of singular simplices $\operatorname{im}(\sigma) \cap \operatorname{Sing}_{n}\left(\mathscr{L}_{\Sigma}(x)\right)$ is locally finite in $\mathscr{L}_{\Sigma}(x)$ for a.e. $x \in X$.

The set of foliated singular $n$-simplices is denoted by $\mathscr{S}_{n}(\Sigma)$.

See Remark 3.11 below for comments.

Remark 3.7. Since the natural measure of the $\left(\operatorname{im}(\sigma), \nu_{t}\right)$-space $A$ in the previous definition is finite, a.e. fiber of $\sigma: A \rightarrow \mathscr{R} \backslash \operatorname{Sing}_{n}(\Sigma)$ is finite.

On each fiber we have the usual face operators $\partial_{i}: \operatorname{Sing}_{n}\left(\Sigma_{x}\right) \rightarrow \operatorname{Sing}_{n-1}\left(\Sigma_{x}\right)$ and degeneracy operators $s_{i}: \operatorname{Sing}_{n}\left(\Sigma_{x}\right) \rightarrow \operatorname{Sing}_{n+1}\left(\Sigma_{x}\right)$ for $i \in\{0,1, \ldots, n\}$. They induce $\mathscr{R}$-equivariant maps, denoted by the same symbols, $\partial_{i}: \operatorname{Sing}_{n}(\Sigma) \rightarrow$ $\operatorname{Sing}_{n-1}(\Sigma)$ and $s_{i}: \operatorname{Sing}_{n}(\Sigma) \rightarrow \operatorname{Sing}_{n+1}(\Sigma)$. One immediately sees that $\partial_{i}, s_{i}$ map admissible sets to admissible sets and are Borel on admissible sets.

The set $\left\{\mathscr{S}_{n}(\Sigma)\right\}_{n \geq 0}$ becomes a simplicial set by the face and degeneracy operators. Like for any simplicial set, there is an associated (unnormalized) chain complex $\mathrm{C}_{*}(\Sigma)$ with $\mathrm{C}_{n}(\Sigma)=\mathbb{Z}\left[\mathscr{S}_{n}(\Sigma)\right]$, and its differential $d: \mathrm{C}_{n}(\Sigma) \rightarrow \mathrm{C}_{n-1}(\Sigma)$ is the alternating sum of face operators $d=\sum_{i=0}^{n}(-1)^{i} \partial_{i}$.

Definition 3.8. For a singular foliated $n$-simplex $\sigma$ the function

$$
\omega_{\sigma}: \mathscr{R} \backslash \operatorname{Sing}_{n}(\Sigma) \rightarrow \mathbb{Z}, s \mapsto \# \sigma^{-1}(s),
$$

is called the multiplicity function of $\sigma$. We extend its definition linearly to elements in $\mathbb{Z}\left[\mathscr{S}_{n}(\Sigma)\right]$.

Remark 3.9. Note that $\omega_{\sigma}$ is supported in the admissible set $\operatorname{im}(\sigma)$. On $\operatorname{im}(\sigma)$, the multiplicity function $\omega_{\sigma}$ is only well defined up to null-sets (cf. Remark 3.7). Subsequent constructions that use multiplicity functions are insensitive to null-sets so that we can ignore this ambiguity.

Let $\rho \in \mathrm{C}_{n}(\Sigma)$. By condition 3 in Definition 3.6, the formal sum

$$
\rho(x)=\sum_{s \in \operatorname{Sing}_{n}\left(\mathscr{L}_{\Sigma}(x)\right)} \omega_{\rho}(s) s
$$

lies in $\mathrm{C}_{n}^{\text {lf }}\left(\mathscr{L}_{\Sigma}(x)\right)$, which is the chain group of locally finite chains on $\mathscr{L}_{\Sigma}(x)$, for a.e. $x \in X$. The assignment $\mathrm{C}_{n}(\Sigma) \rightarrow \mathrm{C}_{n}^{\text {lf }}\left(\mathscr{L}_{\Sigma}(x)\right), \rho \mapsto \rho(x)$, is compatible with the boundary operator meaning that the square

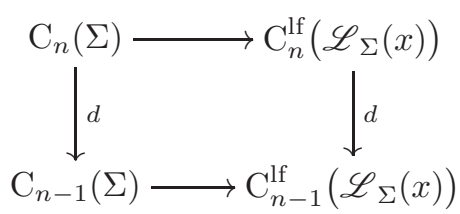

is commutative for a.e. $x \in X$. Hence the chains whose multiplicity function vanishes a.e. form a subcomplex.

Definition 3.10. The singular foliated chain complex $\mathscr{C}_{*}(\Sigma)$ of $\Sigma$ is the quotient of $\mathrm{C}_{*}(\Sigma)$ by the subcomplex of chains whose multiplicity function vanishes almost everywhere. The $n$-th homology of $\mathscr{C}_{*}(\Sigma)$ is denoted by $\mathscr{H}_{n}(\Sigma)$ and called the singular foliated homology of $\Sigma$. 
Remark 3.11. In Definition 3.6, the condition that $\operatorname{im}(\sigma)$ is admissible and on the measures are needed for the approximation results of Lemma 3.24 and Theorem 3.25. Without the condition of locally finiteness, the formal chains in (3.2) do not form a well-defined chain complex, which is essential for defining $\mathscr{C}_{*}(\Sigma)$.

\subsection{Functoriality and homotopy invariance.}

Theorem 3.12. The singular foliated chain complex, thus the singular foliated homology, of $\mathscr{R}$-spaces is functorial with respect to geometric $\mathscr{R}$-maps.

Proof. Let $\phi: \Sigma \rightarrow \Phi$ be a geometric $\mathscr{R}$-map. Since $\phi$ is fiberwise continuous, it induces a map $\operatorname{Sing}_{n}(\phi): \operatorname{Sing}_{n}(\Sigma) \rightarrow \operatorname{Sing}_{n}(\Phi)$, which descends to a map on the quotients $\mathscr{R} \backslash \operatorname{Sing}_{n}(\phi): \mathscr{R} \backslash \operatorname{Sing}_{n}(\Sigma) \rightarrow \mathscr{R} \backslash \operatorname{Sing}_{n}(\Phi)$. Let $\sigma: A \rightarrow \mathscr{R} \backslash \operatorname{Sing}_{n}(\Sigma)$ be a singular foliated $n$-simplex. We have to show that $\left(\mathscr{R} \backslash \operatorname{Sing}_{n}(\phi)\right) \circ \sigma$ is a singular foliated $n$-simplex.

Set $V=\operatorname{im}(\sigma)$, and let $\bar{V}$ denote the pullback of $V$ to $\operatorname{Sing}_{n}(\Sigma)$. The set $V$ is admissible. Moreover, $\bar{V}$ is an $(X, \mu)$-space and carries the natural measure $\alpha=\nu^{\mu}$ (see Definition 2.4). Let $W=\left(\mathscr{R} \backslash \operatorname{Sing}_{n}(\phi)\right)(V)$, and let $\bar{W}$ be the pullback of $W$ to $\operatorname{Sing}_{n}(\Phi)$. By Lemma 3.5, $W$ and $\bar{W}$ are admissible, and $\operatorname{Sing}_{n}(\phi): \bar{V} \rightarrow \bar{W}$ is a map of $X$-spaces. Let $\beta=\nu^{\mu}$ be the natural measure of $\bar{W}$ as an $(X, \mu)$-space. Further, let $\alpha_{t}, \beta_{t}$ be the transversal measures of $\alpha, \beta$, respectively. Viewing $\bar{V}$ as an $(\bar{W}, \beta)$-space via $\operatorname{Sing}_{n}(\phi)$ and, similarly, $V$ as an $\left(W, \beta_{t}\right)$-space, consider the natural measures $\nu^{\beta}, \nu^{\beta_{t}}$ on $\bar{V}, V$ respectively. Since $\left.\operatorname{Sing}_{n}(\phi)\right|_{V}$ is a map of $X$ spaces, $\alpha=\nu^{\beta}$. The pullback of an $\mathscr{R}$-fundamental domain of $W$ to $V$ is one of $V$, hence $\alpha_{t}=\nu^{\beta_{t}}$. Let $\lambda$ be the Lebesgue measure of $A$. By definition of a singular foliated simplex, $\lambda=\nu^{\alpha_{t}}$. Natural measures are transitive in the sense that the natural measure $\lambda=\nu^{\alpha_{t}}=\nu^{\left(\nu_{t}^{\beta}\right)}$ on $A$ as an $V$-space via $\sigma$ equals the natural measure $\nu^{\beta_{t}}$ of $A$ as an $W$-space via $\left(\mathscr{R} \backslash \operatorname{Sing}_{n}(\phi)\right) \circ \sigma$. Thus, $\lambda=\nu^{\beta_{t}}$ as desired. Since $\phi$ is proper on the fibers, the quotient map $\mathscr{R} \backslash \Sigma \rightarrow \mathscr{R} \backslash \Phi$ is proper on leaves; hence $\left(\mathscr{R} \backslash \operatorname{Sing}_{n}(\phi)\right) \circ \sigma$ satisfies the third property of Definition 3.6.

To sum up, $\phi$ induces a well-defined map $\mathscr{S}_{n}(\Sigma) \rightarrow \mathscr{S}_{n}(\Phi)$ and, by linear extension, $\mathrm{C}_{n}(\Sigma) \rightarrow \mathrm{C}_{n}(\Phi)$. The latter descends to a map $\mathscr{C}_{n}(\Sigma) \rightarrow \mathscr{C}_{n}(\Phi)$, which follows from the following observation, which we record in a Remark for later reference.

Remark 3.13. The assignment $\mathrm{C}_{n}(\Sigma) \rightarrow \mathrm{C}_{n}^{\mathrm{lf}}\left(\mathscr{L}_{\Sigma}(x)\right), \rho \mapsto \rho(x)$ in (3.2) descends to $\mathscr{C}_{n}(\Sigma) \rightarrow \mathrm{C}_{n}^{\mathrm{lf}}\left(\mathscr{L}_{\Sigma}(x)\right)$. Let $\phi: \Sigma \rightarrow \Phi$ be a geometric $\mathscr{R}$-map. Then one easily verifies that the induced map $\mathscr{C}_{n}(\phi)$ is compatible with $\rho \mapsto \rho(x)$ in the sense that the square (cf. (3.3))

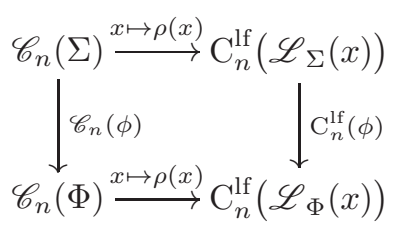

commutes for a.e. $x \in X$.

Next we present (in an informal way) a general principle that allows to transfer proofs from ordinary homology to singular foliated homology.

Remark 3.14 (Extension principle). Let $F_{*}$ be a functor

$$
F_{*}:\{\text { top. spaces }\} \rightarrow\{\text { chain complexes }\}
$$

where $F_{*}(Y)$ is $\mathrm{C}_{*}(Y), \mathrm{C}_{*}(Y \times[0,1])$ or $\mathrm{C}_{*+1}(Y)$. Instead of formalizing the extension principle in greater generality, we stick to these cases. Let $G_{*}$ be another such functor. Suppose there is natural transformation $\omega_{*}: F_{*} \rightarrow G_{*}$. Let $F_{*}^{\text {lf }}$ and $G_{*}^{\text {lf }}$ be the locally finite versions of $F_{*}$ and $G_{*}$, respectively: If e.g. $F_{*}(Y)=\mathrm{C}_{*}(Y)$, 
then $F_{*}^{\mathrm{lf}}(Y)=\mathrm{C}_{*}^{\mathrm{lf}}(Y)$. By naturality we have a commutative diagram for a singular $p$-simplex $s: \Delta^{p} \rightarrow Y$ :

$$
\begin{gathered}
F_{p}(Y) \stackrel{\omega_{p}(Y)}{\longrightarrow} G_{p}(Y) \\
F_{p}(s) \uparrow \begin{array}{r}
G_{p}(s) \\
F_{p}\left(\Delta^{p}\right)
\end{array} \\
\stackrel{\omega_{p}\left(\Delta^{p}\right)}{\longrightarrow} G_{p}\left(\Delta^{p}\right)
\end{gathered}
$$

If e.g. $F_{p}(Y)=G_{p}(Y)=\mathrm{C}_{p}(Y)$, we have $\omega_{p}(Y)(s)=\mathrm{C}_{p}(s)\left(\omega_{p}\left(\Delta^{p}\right)\left(\operatorname{id}_{\Delta^{p}}\right)\right)$. So "supports of simplices are not enlarged". As a consequence (similar for all other examples of $F$ and $G), \omega_{*}$ naturally extends to $F_{*}^{\text {lf }} \rightarrow G_{*}^{\text {lf }}$. Now let

$\mathscr{F}, \mathscr{G}:\{\mathscr{R}$-spaces $\} \rightarrow\{$ chain complexes $\}, i \in\{0,1\}$,

be the corresponding versions of $F$ and $G$, respectively, for $\mathscr{R}$-spaces, that is, if e.g. $F(Y)=\mathrm{C}_{*}(Y)$, then $\mathscr{F}(\Sigma)=\mathscr{C}_{*}(\Sigma)$ for an $\mathscr{R}$-space $\Sigma$.

Then $\omega$ gives rise to a natural transformation $\Omega: \mathscr{F} \rightarrow \mathscr{G}$ such that for every $\mathscr{R}$-space $\Sigma$ the diagram

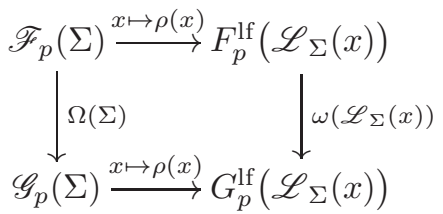

commutes for a.e. $x \in X$ and all $p \geq 0$.

We illustrate the idea for the case $F_{p}(Y)=G_{p}(Y)=\mathrm{C}_{p}(Y)$. Pick $a_{1}, \ldots, a_{m} \in \mathbb{Z}$ and $s_{1}, \ldots, s_{m} \in \operatorname{Sing}_{p}\left(\Delta^{p}\right)$ such that $\omega_{p}\left(\Delta^{p}\right)=\sum_{i=1}^{m} a_{i} s_{i}$. Let $\Sigma$ be an $\mathscr{R}$-space and $\sigma: A \rightarrow \mathscr{R} \backslash \operatorname{Sing}_{p}(\Sigma)$ be a foliated singular $p$-simplex. Then $\Omega$ is defined by $\Omega_{p}(\Sigma)(\sigma)=\sum_{i=1}^{m} a_{i} \rho_{i}$ where $\rho_{i}: A \rightarrow \mathscr{R} \backslash \operatorname{Sing}_{p}(\Sigma)$ is given by the composition

$$
A \stackrel{\sigma}{\rightarrow} \mathscr{R} \backslash \operatorname{Sing}_{p}(\Sigma) \stackrel{s \mapsto s \circ s_{i}}{\longrightarrow} \mathscr{R} \backslash \operatorname{Sing}_{p}(\Sigma) .
$$

Theorem 3.15. Geometric $\mathscr{R}$-maps that are geometrically $\mathscr{R}$-homotopic induce the same map in singular foliated homology.

Proof. It suffices to show that the two inclusion maps $i_{0}, i_{1}: \Sigma \rightarrow \Sigma \times[0,1]$ given by $i_{0}(x)=(x, 0)$ and $i_{1}(x)=(x, 1)$ induce chain homotopic maps on the singular foliated chain complexes.

By [37, Theorem 3 on p. 174] there is natural transformation $D_{*}: F_{*} \rightarrow G_{*}$ of the functors $F_{p}(Y)=\mathrm{C}_{p}(Y)$ and $G_{p}(Y)=\mathrm{C}_{p+1}(Y \times[0,1])$ such that $D_{*}(Y)$ is a chain homotopy between the chain maps induced by $i_{0}$ and $i_{1}$. An application of the extension principle yields a corresponding chain homotopy for the singular foliated chain complexes.

3.4. Support mass. Throughout, let $\Sigma$ be an $\mathscr{R}$-space. By Definition 3.6, the $\operatorname{support} \operatorname{supp}\left(\omega_{\rho}\right) \subset \mathscr{R} \backslash \operatorname{Sing}_{n}(\Sigma)$ of the multiplicity function $\omega_{\rho}$ (Definition 3.8) of an element $\rho \in \mathscr{C}_{n}(\Sigma)$ is admissible, and $\omega_{\rho}$ is integrable with respect to the transversal measure $\nu_{t}$ (Definition 3.4).

Definition 3.16. The support mass of $\rho \in \mathscr{C}_{n}(\Sigma)$ is defined as

$$
\operatorname{mass}(\rho)=\nu_{t}\left(\operatorname{supp}\left(\omega_{\rho}\right)\right) \text {. }
$$

Note that the support mass of a foliated singular simplex $\sigma: A \rightarrow \mathscr{R} \backslash \operatorname{Sing}_{n}(\Sigma)$ is finite since $\operatorname{mass}(\sigma) \leq \lambda(A)<\infty$ by Definition 3.6.

Lemma 3.17. For all $\rho, \rho^{\prime} \in \mathscr{C}_{n}(\Sigma)$ we have $\operatorname{mass}\left(\rho+\rho^{\prime}\right) \leq \operatorname{mass}(\rho)+\operatorname{mass}\left(\rho^{\prime}\right)$. Proof. This follows from $\operatorname{supp}\left(\omega_{\rho+\rho^{\prime}}\right)=\operatorname{supp}\left(\omega_{\rho}+\omega_{\rho^{\prime}}\right) \subset \operatorname{supp}\left(\omega_{\rho}\right) \cup \operatorname{supp}\left(\omega_{\rho^{\prime}}\right)$. 
Note that the support mass is subadditive but not a norm since mass $(d \rho)=$ $\operatorname{mass}(\rho)$ for $\rho \in \mathscr{C}_{n}(\Sigma)$ and $d \in \mathbb{Z}$.

Definition 3.18. The support mass of a homology class in $\mathscr{H}_{n}(\Sigma)$ is the infimum of support masses of its representing cycles. We use the same notation mass $(\kappa)$ for the support mass of a homology class $\kappa$.

Remark 3.19. Let $\Phi$ be an $\mathscr{R}$-simplicial complex, and let $\rho \in \mathscr{C}_{n}(|\Phi|)$. Assume that $\omega_{\rho}$ is supported in $\mathscr{R} \backslash \Phi^{(n)} \subset \mathscr{R} \backslash \operatorname{Sing}_{n}(|\Phi|)$ (for this inclusion see Remark 3.1). Then we have

$$
\operatorname{mass}(\rho) \leq \nu_{t}\left(\mathscr{R} \backslash \Phi^{(n)}\right)=(n+1) ! \cdot \sigma_{n}(\Phi) .
$$

Lemma 3.20. The homomorphisms $\mathscr{C}_{*}(\phi)$ and $\mathscr{H}_{*}(\phi)$ that are induced by a geometric $\mathscr{R}$-map $\phi$ do not increase the support mass.

Proof. Let $\phi: \Sigma \rightarrow \Phi$ be a geometric $\mathscr{R}$-map and $\rho \in \mathscr{C}_{n}(\Sigma)$. Let $\rho^{\prime}=\mathscr{C}_{n}(\phi)(\rho)$. We need only show that

$$
\nu_{t}\left(\operatorname{supp}\left(\omega_{\rho^{\prime}}\right)\right) \leq \nu_{t}\left(\operatorname{supp}\left(\omega_{\rho}\right)\right) .
$$

First one verifies leafwise using diagram (3.4) that

$$
\operatorname{supp}\left(\omega_{\rho^{\prime}}\right) \subset\left(\mathscr{R} \backslash \operatorname{Sing}_{n}(\phi)\right)\left(\operatorname{supp}\left(\omega_{\rho}\right)\right) .
$$

Then (3.7) is a consequence of the following general fact about transversal measures: By changing notation, let $\Sigma$ and $\Phi$ denote $(X, \mu)$-spaces with discrete $\mathscr{R}$-actions, and let $\phi: \Sigma \rightarrow \Phi$ be an $\mathscr{R}$-equivariant map of $X$-spaces. Let $A \subset \mathscr{R} \backslash \Sigma$ be a Borel subset. Then

$$
\nu_{t}((\mathscr{R} \backslash \phi)(A)) \leq \nu_{t}(A) .
$$

The corresponding assertion $\nu(\phi(B)) \leq \nu(B)$ for $B \subset \Sigma$ and the natural measure $\nu$ is clear from Definition 2.4. Now (3.9) follows from the fact that if $\mathscr{F}$ is a fundamental domain for $\Phi$, then $\phi^{-1}(\mathscr{F})$ is one for $\Sigma$.

Lemma 3.21. Let $\Sigma, \Phi$ and $\phi$ as in Lemma 2.23. Let $\kappa \in \mathscr{C}_{n}(|\Sigma|)$ be a cycle such that $\omega_{\kappa}$ is supported in $\mathscr{R} \backslash \Sigma^{(n)}$. Then $\mathscr{H}_{n}(|\phi|)([\kappa]) \in \mathscr{H}_{n}(|\Phi|)$ has support mass at most $(n+1) !^{2} \cdot \sigma_{n}(\Phi)$.

Proof. For every topological space $N$, there is chain map $Y_{*}(N): \mathrm{C}_{*}(N) \rightarrow \mathrm{C}_{*}(N)$ that realizes barycentric subdivision [6, p. 224]; $Y_{*}$ is natural in $N$ and thus a natural transformation in the sense of Remark 3.14. The elementary properties of $Y_{*}$ are $[6$, p. 225]:

a) Let $\sigma: \Delta^{p} \rightarrow N$ be degenerate in the sense that there is a surjective affine and vertex-preserving map $\alpha: \Delta^{p} \rightarrow \Delta^{q}$ with $q<p$ and a singular $q$-simplex $\sigma^{\prime}: \Delta^{q} \rightarrow N$ such that $\sigma=\sigma^{\prime} \circ \alpha$. Then $\left(Y_{p}(N)\right)(\sigma)=0$.

b) $\left(Y_{p}\left(\Delta^{p}\right)\right)\left(\operatorname{id}_{\Delta^{p}}\right)$ is a linear combination of the affine simplices of the barycentric subdivision of $\Delta^{p}$.

c) $Y_{*}$ is naturally chain homotopic to the identity.

The first property is not explicitly stated in [6] but follows from naturality and

$$
\left(Y_{p}\left(\Delta^{q}\right)\right)(\gamma \sigma)=\operatorname{sign}(\gamma)\left(Y_{p}\left(\Delta^{q}\right)\right)(\sigma)
$$

for $\gamma \in \mathbf{S}(q+1), \sigma: \Delta^{p} \rightarrow \Delta^{q}$ and the natural $\mathbf{S}(q+1)$-action on $\Delta^{q}$. As $Y_{p}\left(\Delta^{q}\right)$ is inductively defined [6, p. 224], Equation (3.10) can be easily proved by induction over $p$.

By the extension principle (see Remark 3.14), $Y$ gives rise to a natural transformation $\mathscr{Y}_{*}$ on the singular foliated chain complex. Set $\rho=\mathscr{C}_{n}(|\phi|)(\kappa)$ for $\kappa$ as in the hypothesis. Since $\phi$ is simplicial, we can decompose $\rho$ as $\rho=\rho_{1}+\rho_{2}$ such that $\omega_{\rho_{1}}$ is supported in the degenerate simplices and $\omega_{\rho_{2}}$ is supported in $\mathscr{R} \backslash \Phi^{(n)}$. The 
first and third property of $Y_{*}$ and the analogous one of $\mathscr{Y}_{*}$ imply that the homology classes satisfy

$$
[\rho]=\left[\left(\mathscr{Y}_{n}(|\Phi|)\right)(\rho)\right]=\left[\left(\mathscr{Y}_{n}(|\Phi|)\right)\left(\rho_{2}\right)\right] .
$$

By the second property the multiplicity function of $\left(\mathscr{Y}_{n}(|\Phi|)\right)\left(\rho_{2}\right)$ is supported in $\mathscr{R} \backslash \operatorname{sd}(\Phi)^{(n)} \subset \mathscr{R} \backslash \operatorname{Sing}_{n}(|\Phi|)$. By Remark 3.19 we finally get

$$
\operatorname{mass}([\rho]) \leq(n+1) ! \cdot \sigma_{n}(\operatorname{sd}(\Phi))=(n+1) !^{2} \cdot \sigma_{n}(\Phi)
$$

For the rest of this Subsection retain the setting of Assumption 1.2. The goal is to define a notion of support mass on $\mathrm{H}_{n}^{\Gamma}\left(\widetilde{M} ; \mathrm{L}^{\infty}(X ; \mathbb{Z})\right)$ and to compare it with the one on $\mathscr{H}_{n}(X \times \widetilde{M})$.

Definition 3.22. Let $p: \widetilde{M} \rightarrow M$ be the natural projection. The choice of a $\Gamma$-fundamental domain $\mathscr{F} \subset \operatorname{Sing}_{n}(\widetilde{M})$ gives rise to an isomorphism $\mathrm{C}_{n}(\widetilde{M}) \cong$ $\mathbb{Z} \Gamma \otimes_{\mathbb{Z}} \mathrm{C}_{n}(M)$ of left $\mathbb{Z} \Gamma$-modules that maps $s \in \gamma \mathscr{F}$ to $\gamma \otimes p \circ s$. So we get the isomorphism

$$
\mathrm{L}^{\infty}(X ; \mathbb{Z}) \otimes_{\mathbb{Z}} \mathrm{C}_{n}(M) \cong \mathrm{L}^{\infty}(X ; \mathbb{Z}) \otimes_{\mathbb{Z} \Gamma} \mathrm{C}_{n}(\widetilde{M})
$$

For $s_{1}, \ldots, s_{k} \in \operatorname{Sing}_{n}(M)$ with $s_{i} \neq s_{j}$ for $i \neq j$ and $f_{1}, \ldots, f_{k} \in \mathrm{L}^{\infty}(X ; \mathbb{Z})$ we define

$$
\operatorname{mass}\left(\sum_{i=1}^{k} f_{i} \otimes_{\mathbb{Z}} s_{i}\right):=\sum_{i=1}^{k} \mu\left(\operatorname{supp}\left(f_{i}\right)\right)
$$

as the support mass of the chain $\sum_{i} f_{i} \otimes_{\mathbb{Z}} s_{i}$. The support mass of elements in $\mathrm{L}^{\infty}(X ; \mathbb{Z}) \otimes_{\mathbb{Z} \Gamma} \mathrm{C}_{n}(\widetilde{M})$ is then defined via (3.11). The support mass does not depend on the choice of $\mathscr{F}$. The support mass of a homology class of $\mathrm{H}_{n}^{\Gamma}\left(\widetilde{M} ; \mathrm{L}^{\infty}(X ; \mathbb{Z})\right)$ is defined as the infimum of support masses of representing cycles.

In the sequel we use the term isometric in the sense of preserving support masses.

Lemma 3.23. The chain homomorphism

$$
\lambda_{*}: \mathrm{L}^{\infty}(X ; \mathbb{Z}) \otimes_{\mathbb{Z}} \mathrm{C}_{*}(\widetilde{M}) \rightarrow \mathscr{C}_{*}(X \times \widetilde{M})
$$

that is uniquely determined by

$$
\lambda_{n}\left(\chi_{Y} \otimes s\right)=\left(\sigma: j^{-1}(Y) \rightarrow \Gamma \backslash\left(X \times \operatorname{Sing}_{n}(\widetilde{M})\right)\right) \text { with } \sigma(y)=(j(y), s) .
$$

for $Y \subset X$ Borel, $s \in \operatorname{Sing}_{n}(\widetilde{M})$ and a measure preserving Borel isomorphism $j:[0,1] \rightarrow X$ does not depend on the choice of $j$. Further, $\lambda_{*}$ descends to an isometric map denoted by the same symbol

$$
\lambda_{*}: \mathrm{L}^{\infty}(X ; \mathbb{Z}) \otimes_{\mathbb{Z} \Gamma} \mathrm{C}_{*}(\widetilde{M}) \rightarrow \mathscr{C}_{*}(X \times \widetilde{M}) .
$$

Proof. This is a matter of straightforward verification.

Lemma 3.24. Let $\rho \in \mathscr{C}_{n}(X \times \widetilde{M})$ and $\epsilon>0$. Then there is $c \in \mathrm{L}^{\infty}(X ; \mathbb{Z}) \otimes_{\mathbb{Z} \Gamma}$ $\mathrm{C}_{n}(\widetilde{M})$ such that $\operatorname{mass}\left(\rho-\lambda_{n}(c)\right)<\epsilon$.

Proof. Without loss of generality, we can assume that $\rho: A \rightarrow \mathscr{R} \backslash\left(X \times \operatorname{Sing}_{n}(\widetilde{M})\right)$ is a foliated singular simplex. Let $\mathscr{F} \subset \operatorname{Sing}_{n}(\widetilde{M})$ be a $\Gamma$-fundamental domain. After identifying $X \times \mathscr{F}$ with $\Gamma \backslash\left(X \times \operatorname{Sing}_{n}(\widetilde{M})\right)$, there is a countable set $S=$ $\left\{s_{1}, s_{2}, \ldots\right\} \subset \mathscr{F}$ such that $\operatorname{im}(\rho) \subset X \times S$. For $r, s>0$ set

$$
W_{r, s}=\left\{\left(x, s_{i}\right) ; x \in X, \# \rho^{-1}\left(x, s_{i}\right) \leq r, 1 \leq i \leq s\right\} \subset X \times S .
$$

Let $A_{r, s}=\rho^{-1}\left(W_{r, s}\right)$. The sets $W_{r, s}$ form a directed system whose union is $\operatorname{im}(\rho)$. Thus for sufficiently large $r, s$

$$
\operatorname{mass}\left(\rho-\left.\rho\right|_{A_{r, s}}\right)=\operatorname{mass}\left(\left.\rho\right|_{A-A_{r, s}}\right) \leq \lambda\left(A-A_{r, s}\right) \leq \epsilon,
$$


where $\lambda$ is the Lebesgue measure. It remains to show that $\left.\rho\right|_{A_{r, s}} \in \operatorname{im}\left(\lambda_{n}\right)$. Fix $r, s$. There is a Borel partition $A_{r, s}=\bigcup_{k<r, l<s} B_{k, l}$ such that $\operatorname{im}\left(\left.\sigma\right|_{B_{k, l}}\right) \subset X \times\left\{s_{l}\right\}$ and $\left.\sigma\right|_{B_{k, l}}$ is injective (theorem of selection; see e.g. [33, Lemma 3.1] for the version needed here). In particular, $\left.\sigma\right|_{B_{k, l}}$ is a measure preserving Borel isomorphism $B_{k, l} \rightarrow$ $X_{k, l}=X_{k, l} \times\left\{s_{l}\right\}$ onto its image $X_{k, l}$. The chain

$$
c_{r, s}=\sum_{k \leq r, l \leq s} \chi_{X_{k, l}} \otimes s_{l} \in \mathrm{L}^{\infty}(X ; \mathbb{Z}) \otimes_{\mathbb{Z} \Gamma} \mathrm{C}_{n}(\widetilde{M}) .
$$

satisfies $\lambda_{n}\left(c_{r, s}\right)=\left.\rho\right|_{A_{r, s}}$.

Theorem 3.25. The homomorphism $\mathrm{H}_{n}\left(\lambda_{*}\right): \mathrm{H}_{n}^{\Gamma}\left(\widetilde{M} ; \mathrm{L}^{\infty}(X ; \mathbb{Z})\right) \rightarrow \mathscr{H}_{n}(X \times \widetilde{M})$ is isometric.

Proof. Let $\rho \in \mathscr{C}_{n}(X \times \widetilde{M})$ and $c \in \mathrm{L}^{\infty}(X ; \mathbb{Z}) \otimes_{\mathbb{Z} \Gamma} \mathrm{C}_{n}(\widetilde{M})$. Let $\epsilon>0$. Since $\lambda_{n}$ is isometric, $\mathscr{H}_{n}\left(\lambda_{*}\right)$ does not increase support masses. It remains to show: If $\rho$ and $\lambda_{n}(c)$ are homologous, then there is a chain $c_{1} \in \mathrm{L}^{\infty}(X ; \mathbb{Z}) \otimes_{\mathbb{Z} \Gamma} \mathrm{C}_{n}(\widetilde{M})$ that is homologous to $c$ and satisfies

$$
\operatorname{mass}\left(c_{1}\right)<\operatorname{mass}(\rho)+\epsilon .
$$

Let $\kappa \in \mathscr{C}_{n+1}(X \times \widetilde{M})$ be such that $\rho=\lambda_{n}(c)+d \kappa$. By Lemma 3.24, we find $c_{0}$ with $\operatorname{mass}\left(\lambda_{n+1}\left(c_{0}\right)-\kappa\right)<\epsilon /(n+1)$. Then $c_{1}=c+d c_{0}$ satisfies

$$
\begin{aligned}
\operatorname{mass}\left(c_{1}\right) & =\operatorname{mass}\left(\lambda_{n}\left(c_{1}\right)\right) \\
& \leq \operatorname{mass}(\rho)+\operatorname{mass}\left(d\left(\lambda_{n+1}\left(c_{0}\right)-\kappa\right)\right) \\
& \leq \operatorname{mass}(\rho)+(n+1) \operatorname{mass}\left(\lambda_{n+1}\left(c_{0}\right)-\kappa\right)<\operatorname{mass}(\rho)+\epsilon .
\end{aligned}
$$

3.5. Fundamental classes. We retain the setting of Assumption 1.3.

Remark 3.26 (Fundamental classes). Let

$$
j_{*}: \mathrm{C}_{*}(M)=\mathbb{Z} \otimes_{\mathbb{Z} \Gamma} \mathrm{C}_{*}(\widetilde{M}) \rightarrow \mathrm{L}^{\infty}(X ; \mathbb{Z}) \otimes_{\mathbb{Z} \Gamma} \mathrm{C}_{*}(\widetilde{M})
$$

be the map coming from inclusion of constant functions. Let $\lambda_{*}$ be the map from Lemma 3.23. Let $[M] \in \mathrm{H}_{n}(M)$ be the fundamental class of $M$. By convention, the images $\mathrm{H}_{n}\left(j_{*}\right)([M]) \in \mathrm{H}_{n}^{\Gamma}\left(\widetilde{M} ; \mathrm{L}^{\infty}(X ; \mathbb{Z})\right)$ and $H_{n}\left(\lambda_{*} \circ j_{*}\right)([M]) \in \mathscr{H}_{n}(X \times \widetilde{M})$ are also called fundamental classes.

Moreover, $\mathrm{H}_{n}\left(j_{*}\right)$ is an isomorphism if and only if the $\Gamma$-action on $(X, \mu)$ is ergodic: By equivariant Poincaré duality,

$$
\mathrm{H}_{n}^{\Gamma}\left(\widetilde{M} ; \mathrm{L}^{\infty}(X ; \mathbb{Z})\right) \cong \mathrm{H}_{\Gamma}^{0}\left(\widetilde{M} ; \mathrm{L}^{\infty}(X ; \mathbb{Z})\right) \cong \mathrm{L}^{\infty}(X ; \mathbb{Z})^{\Gamma}
$$

and $\mathrm{H}_{n}\left(j_{*}\right)$ corresponds to the inclusion $\mathbb{Z} \rightarrow \mathrm{L}^{\infty}(X ; \mathbb{Z})^{\Gamma}$ of constant functions under this isomorphism.

Remark 3.27 (Foliated simplicial volume). We point out the relation to Connes's simplicial volume for foliations. Suppose for the moment that the $\mathscr{C}_{n}(\Sigma)$ is defined by chains with real coefficients, that is, as the quotient of $\mathbb{R}\left[\mathscr{S}_{n}(\Sigma)\right]$ by the chains with a.e. vanishing multiplicity function. Consider the following norm on $\mathscr{C}_{n}(\Sigma)$

$$
|\rho|=\int_{\mathscr{R} \backslash \operatorname{Sing}_{n}(\Sigma)}\left|\omega_{\rho}\right| .
$$

By Definition 3.6, the norm $|\sigma|$ of a foliated singular simplex $\sigma: A \rightarrow \mathscr{R} \backslash \operatorname{Sing}_{n}(\Sigma)$ equals the Lebesgue measure $\lambda(A)$ of $A$. The norm on $\mathscr{C}_{n}(\Sigma)$ induces a semi-norm on $\mathscr{H}_{n}(\Sigma)$ by taking the infimum of representing chains. Let $M$ be as in the previous remark. The norm of the fundamental class in $\mathscr{H}_{n}(X \times \widetilde{M})$ is the foliated simplicial volume of the measured foliation $\Gamma \backslash(X \times \widetilde{M})$, which is attributed to Connes and described by Gromov in [20, section 2.4.B]. 


\section{Proof of Theorem A}

4.1. $\mathscr{R}$-covers with controlled multiplicity. In this section we construct the $\mathscr{R}$-cover needed in the proof of Theorem A following the strategy in Section 1.3. By convention, if $B(R)$ denotes a metric ball of radius $R$ in a metric space, then $B\left(R^{\prime}\right)$ denotes the concentric ball of radius $R^{\prime}$.

Theorem 4.1. Let $M$ be a Riemannian manifold and $(X, \mu)$ be a probability $\Gamma$ space as in Assumption 1.3. Assume further the following packing inequality: There is a constant $N_{0} \in \mathbb{N}$ such that each ball of radius 1 in $\widetilde{M}$ contains at most $N_{0}$ disjoint balls of radius $1 / 16$. Then there are countable families $\left\{A_{i}\right\}_{i \in I}$ of Borel subsets of $X$ and $\left\{B_{i}(3 / 16)\right\}_{i \in I}$ of balls of radius $3 / 16$ in $\widetilde{M}$ such that

a) $\mathscr{U}(3 / 16):=\left\{A_{i} \times B_{i}(3 / 16)\right\}_{i \in I}$ is an $\mathscr{R}$-covering of $X \times \widetilde{M}$,

b) $\mathscr{U}(1 / 4)_{x}:=\left\{B_{i}(1 / 4) ; x \in A_{i}\right\}_{i \in I}$ has multiplicity at most $N_{0}$ for a.e. $x \in X$.

For the proof we need the following easy lemma.

Lemma 4.2. Let $(X, \mu)$ and $\Gamma$ be as in Assumption 1.1. Let $\gamma \in \Gamma \backslash\{1\}$. Let $A \subset X$ be a Borel set with $\mu(A)>0$. Then there is a Borel subset $A^{\prime} \subset A$ with $\mu\left(A^{\prime}\right)>0$ such that $\mu\left(\gamma A^{\prime} \cap A^{\prime}\right)=0$.

Proof. By [38, Theorem 3.2], $X$ is equivariantly Borel isomorphic to a $\Gamma$-invariant Borel subset of a compact metric space $Y$ with a continuous $\Gamma$-action. Thus we may assume that $X \subset Y$, and the measure $\mu$ is extended to $Y$ by zero. By Ulam's theorem [9, Proposition 8.1.10 on p. 258], $\mu$ is regular on $Y$.

Upon subtracting a null set from $A$, we can assume that $\gamma x \neq x$ for all $x \in A$. Next we show that there exists $x_{0} \in A$ such that $\mu(A \cap U)>0$ for every open neighborhood $U$ of $x_{0}$. Arguing by contradiction, suppose that every $x \in A$ has an open neighborhood $U_{x}$ with $\mu\left(A \cap U_{x}\right)=0$. This yields $\mu(K)=0$ for every compact $K \subset A$, thus $\mu(A)=0$ by regularity. Let $U, V$ be disjoint, open neighborhoods of $x_{0}$ and $\gamma x_{0}$, respectively, such that $\gamma U \subset V$. Then $A^{\prime}:=A \cap U$ satisfies the conclusion.

Proof of Theorem 4.1. An $\mathscr{R}$-packing by balls of radius $r$ is, by definition, an $\mathscr{R}$ packing whose sets are of the type $A \times B$ where $B$ is a ball of radius $r$. We say that an $\mathscr{R}$-packing $\mathscr{U}=\left\{A_{i} \times B_{i}(1 / 16)\right\}_{i \in I}$ of $X \times \widetilde{M}$ by balls of radius $1 / 16$ is non-equivariantly maximal if there does not exist a Borel subset $A \subset X$ with $\mu(A)>0$ and a ball $B(1 / 16) \subset \widetilde{M}$ of radius $1 / 16$ such that

$$
A \times B(1 / 16) \subset X \times \widetilde{M} \backslash \bigcup_{i \in I} A_{i} \times B_{i}(1 / 16)
$$

First claim: For every $A \subset X$ with $\mu(A)>0$ and every finite $F \subset \Gamma$ with $1 \notin F$ there exists $A^{\prime} \subset A$ with $\mu\left(A^{\prime}\right)>0$ such that $\mu\left(A^{\prime} \cap \gamma A^{\prime}\right)=0$ for all $\gamma \in F$.

Let $F=\left\{\gamma_{1}, \ldots, \gamma_{m}\right\}$. Apply Lemma 4.2 repeatedly to obtain Borel sets $A_{1}, \ldots, A_{m}$ of positive measure such that $A_{i+1} \subset A_{i}$ and $\mu\left(A_{i} \cap \gamma_{i} A_{i}\right)=0$. Set $A^{\prime}:=A_{m}$.

Second claim: If $\mathscr{U}$ is not non-equivariantly maximal, then there exists a subset $A^{\prime} \subset X$ of positive measure and a ball $B(1 / 16)$ of radius $1 / 16$ such that

$$
A^{\prime} \times B(1 / 16) \subset X \times \widetilde{M} \backslash \bigcup_{i \in I} A_{i} \times B_{i}(1 / 16),
$$

and $\mathscr{U} \cup\left\{\gamma A^{\prime} \times \gamma B(1 / 16)\right\}_{\gamma \in \Gamma}$ is still an $\mathscr{R}$-packing.

Pick $A \times B(1 / 16)$ as in (4.1). The set

$$
\Gamma_{B}=\{\gamma \in \Gamma ; \gamma \neq 1, \gamma B(1 / 16) \cap B(1 / 16) \neq \emptyset\}
$$


is finite since $\Gamma$ acts properly on $\widetilde{M}$. Now $A^{\prime}$ is obtained from the first claim with $F=\Gamma_{B}$

The set of $\mathscr{R}$-packings on $X \times \widetilde{M}$ by balls of radius $1 / 16$ is partially ordered as follows: $\mathscr{U} \leq \mathscr{V}$ if and only if for every $A \times B \in \mathscr{U}$ there exists $A^{\prime} \subset X$ Borel such that $A=A^{\prime}$ up to null-sets and $A^{\prime} \times B \in \mathscr{V}$.

Let $\left\{\mathscr{U}_{k}\right\}_{k \in K}$ be a totally ordered family of $\mathscr{R}$-packings by balls of radius $1 / 16$. Let $\mathscr{U}_{k}=\left\{A_{i} \times B_{i}\right\}_{i \in I_{k}}$. There exists an upper bound $\mathscr{U}$ of $\left\{\mathscr{U}_{k}\right\}_{k \in K}$ : Set $I:=$ $\coprod_{k \in K} I_{k}$ (disjoint union). For $i, j \in I$, say $i \sim j$ if and only if $A_{i}=A_{j}$ up to null sets and $B_{i}=B_{j}$. Let $J$ be a $\Gamma$-invariant, complete set of $\sim$-representatives. Then $\mathscr{U}=\left\{A_{j} \times B_{j}\right\}_{j \in J}$ is an $\mathscr{R}$-packing and an upper bound of $\left\{\mathscr{U}_{k}\right\}_{k \in K}$.

By Zorn's lemma there exists a maximal element $\mathscr{U}(1 / 16)=\left\{A_{j} \times B_{j}(1 / 16)\right\}_{j \in J}$ (now a different $J$ ). By the second claim, $\mathscr{U}(1 / 16)$ is non-equivariantly maximal. We may and will assume that $\mu\left(A_{j}\right)>0$ for every $j \in J$. By Lemma 2.29, $J$ is countable.

It remains to show that $\mathscr{U}(3 / 16)$ and $\mathscr{U}(1 / 4)$ have the stated properties. If there exist $m \in \widetilde{M}$ and $A \subset X$ with $\mu(A)>0$ such that

$$
(x, m) \notin \bigcup_{j \in J} A_{j} \times B_{j}(3 / 16)
$$

for $x \in A$, then

$$
A \times B(m, 1 / 16) \cap \bigcup_{j \in J} A_{j} \times B_{j}(1 / 16)=\emptyset
$$

for the ball $B(m, 1 / 16)$ of radius $1 / 16$ around $m$ contradicting non-equivariant maximality. It immediately follows from the packing inequality on $\widetilde{M}$ and the fact that $\mathscr{U}(1 / 16)_{x}$ is a packing for a.e. $x \in X$ (Lemma 2.29) that $\mathscr{U}(1 / 4)_{x}$ has multiplicity at most $N_{0}$ for a.e. $x \in X$.

\subsection{The map to the nerve.}

Assumption for Subsection 4.2. Retain the setting of Assumption 1.3. Further, we assume throughout this section:

- Let $M$ be equipped with a Riemannian metric such that the induced metric on the universal cover $\widetilde{M}$ satisfies the following packing inequality: There is a constant $N_{0}$ such that for $r<1$ each ball of radius 1 in $\widetilde{M}$ contains at most $N_{0} r^{-n}$ balls of radius $r$.

- Let $\mathscr{U}=\mathscr{U}(1 / 4)=\left\{A_{i} \times B_{i}(1 / 4)\right\}_{i \in I}$ be the $\mathscr{R}$-cover constructed in Theorem 4.1, which has multiplicity $\leq N_{0}$ (in fact, $\leq 16^{-n} N_{0}$ ).

- We regard the nerve $\mathscr{N}(\mathscr{U})$ as an $\mathscr{R}$-simplicial complex, and we write $|\mathscr{N}(\mathscr{U})|$ for the corresponding $\mathscr{R}$-space (realization), which we equip with length metric (see Remark 2.16). Recall that there is a standard embedding $\mathscr{N}(\mathscr{U}) \subset X \times \Delta(I)$ (see Remark 2.31).

We now define a geometric $\mathscr{R}$-map $\phi: X \times \widetilde{M} \rightarrow|\mathscr{N}(\mathscr{U})|$ such that $\phi_{x}$ is induced by a partition of unity of $\mathscr{U}_{x}$ for a.e. $x \in X$. For $i \in I$ define $\phi_{i}: \widetilde{M} \rightarrow[0,1]$ by

$$
\phi_{i}(m)= \begin{cases}1 & \text { if } m \in B_{i}(3 / 16) \\ 1-16 d\left(m, B_{i}(3 / 16)\right) & \text { if } m \in B_{i}(1 / 4) \backslash B_{i}(3 / 16) \\ 0 & \text { if } m \notin B_{i}(1 / 4),\end{cases}
$$

and let

$$
\phi(x, m)=\left(x, \frac{1}{\sum_{i \in I} \chi_{A_{i}}(x) \phi_{i}(m)} \sum_{i \in I} \chi_{A_{i}}(x) \phi_{i}(m) i\right),
$$


where $\chi_{A_{i}}$ denotes the characteristic function of $A_{i}$. Since $\mathscr{U}_{x}$ is locally finite for a.e. $x \in X, \phi_{x}$ is proper. Lemma $2.28 \mathrm{~b}$ ) implies that $\phi$ is of countable variance, and equivariance of $\phi$ is obvious.

The goal of this subsection is to show the following theorem whose proof is given after a sequence of lemmas.

Theorem 4.3. Let $\phi$ be the map in (4.2). There is a constant const $_{n, N_{0}}$ that only depends on $n$ and $N_{0}$ (but not on $M$ ) such that the image under $\mathscr{H}_{n}(\phi)$ of the fundamental class in $\mathscr{H}_{n}(X \times \widetilde{M})$ (cf. Remark 3.26) has support mass at most const $_{N_{0}, n} \operatorname{vol}(M)$.

Lemma 4.4. For every $k \geq 0$ we have $\sigma_{k}(\mathscr{N}(\mathscr{U}))<\infty$ (cf. Definition 2.9).

Proof. By compactness of $M$, we may choose a complete set $I^{\prime} \subset I$ of $\Gamma$-representatives such that $\bigcup_{i \in I^{\prime}} B_{i}(1 / 4)$ is relatively compact. Let $K$ be the compact closure of $\bigcup_{i \in I^{\prime}} B_{i}(3 / 4)$. Let be $\mathscr{F}$ as in Equation $(2.5)$ in the proof of Lemma 2.34 with $U_{i}=B_{i}(1 / 4)$. If $\left(x, i_{0}, \ldots, i_{k}\right) \in \mathscr{F}_{x}$, then $B_{i_{l}}(1 / 4) \subset K$ for $l \in\{1, \ldots, k\}$. Since the sets $B_{i_{l}}(1 / 16)$ are disjoint (by the proof of Theorem 4.1) and there is an upper bound for the number of disjoint balls of radius $1 / 16$ that are contained in $K$, there is a constant $0<C<\infty$ such $\# \mathscr{F}_{x}<C$ for a.e. $x \in X$. Then Equation (2.6) yields the conclusion.

Lemma 4.5. There is a constant const $_{N_{0}}$ that only depends on $N_{0}$ such that the map $\phi_{x}$ from (4.2) has Lipschitz constant $\leq$ const $_{N_{0}}$ for a.e. $x \in X$.

Proof. For a.e. $x \in X, \phi_{x}$ is the map from $\widetilde{M}$ to the at most $N_{0}$-dimensional nerve of $\mathscr{U}_{x}$ induced by the partition of unity $\left\{\phi_{i} ; i \in I, x \in A_{i}\right\}$. A standard computation [2, Lemma 4.6; 3, Proposition 1] shows that $\phi_{x}$ has a Lipschitz constant bounded in terms of $N_{0}$ and the Lebesgue number of $\mathscr{U}_{x}$ (=1/16 in our case).

Definition 4.6. Let $\Sigma$ be an $\mathscr{R}$-simplicial complex and $s \in \Sigma^{(k)}$. We let $|s| \subset|\Sigma|$ denote the geometric $k$-simplex corresponding to the (combinatorial) $k$-simplex $s \in \Sigma^{(k)}$. The interior of $|s|$ is denoted by $\mid \stackrel{s}{\text {. }}$.

For the proof of Theorem 4.3 we need the following general definition and lemma.

Definition 4.7. Let $\epsilon \geq 0$. Let $\omega:|\Phi| \rightarrow|\Psi|$ be an geometric $\mathscr{R}$-map. Let $\Sigma \subset \Psi^{(k)}$ be an $\mathscr{R}$-invariant Borel subset. A family $\left\{P^{s}\right\}_{s \in \Sigma}$ of points $P^{s} \in|\dot{s}|$ is called a family of $\epsilon$-projectors for $\omega$ if the following conditions hold:

a) $\left\{P^{s}\right\}_{s \in \Sigma} \subset|\Psi|$ is admissible.

b) distance $d\left(P^{s}, \operatorname{im}(\omega) \cap|s|\right)>\epsilon$ for a.e. $s \in \Sigma$.

c) $\left\{P^{s}\right\}_{s \in \Sigma} \subset|\Psi|$ is an $\mathscr{R}$-invariant subset.

For a) notice that $\left\{P^{s}\right\}$ can be seen as a subset of $\operatorname{Sing}_{0}(|\Psi|)$ and admissible is understood in the sense of Definition 3.2. In b) we set $d\left(P_{x}^{s}, \emptyset\right)=\infty$.

Lemma 4.8. Let $\omega:|\Phi| \rightarrow\left|\Psi^{(k)}\right|$ be a geometric $\mathscr{R}$-map that lands in the $k$ skeleton, and let $\Sigma \subset \Psi^{(k)}$ be an $\mathscr{R}$-invariant Borel subset. Let $\left\{P^{s}\right\}_{s \in \Sigma}$ be a family of $\epsilon$-projectors for $\omega$. Define $\psi:|\Phi| \rightarrow|\Psi|$ to be the map obtained from $\omega$ by postcomposition with the radial projections (within $|s|$ ) from $P^{s}$ to the boundary $\partial|s|$ for every $s \in \Sigma$. We say that $\psi$ is obtained from $\omega$ via $\left\{P_{x}^{s}\right\}$. Then the following holds or holds a.e., respectively.

a) $\psi$ is a geometric $\mathscr{R}$-map.

b) Assume that $\epsilon>0$. If $\omega_{x}$ has a Lipschitz constant $C>0$, then $\psi_{x}$ has a Lipschitz constant $C / \epsilon$.

c) The $\psi_{x}$-preimage of the open star of any vertex is contained in the corresponding $\omega_{x}$-preimage. 
d) There is geometric $\mathscr{R}$-homotopy between $\omega$ and $\psi$.

Proof. The proof that $\psi$ is of countable variance is straightforward and follows from the fact that $\left\{P^{s}\right\}$ is admissible. It is clear that $\psi$ is $\mathscr{R}$-equivariant and continuous on fibers. Since $\psi_{x}$ is an approximation of $\omega_{x}$, Lemma 2.24 implies that $\psi$ is a geometric $\mathscr{R}$-map, which is geometrically $\mathscr{R}$-homotopic to $\omega$. For the same reason assertion c) is true. The radial projections $|s| \backslash B_{\epsilon}\left(P^{s}\right) \rightarrow \partial|s| \subset|s|$ have Lipschitz constant $\leq \epsilon^{-1}$. Using the fact that the metric on $|\Psi|$ is a length metric, one easily sees that $\psi_{x}$ has Lipschitz constant $\leq C / \epsilon$ for a.e. $x \in X$.

Lemma 4.9. Let $\omega: X \times \widetilde{M} \rightarrow\left|\mathscr{N}(\mathscr{U})^{(k)}\right|$ be a geometric $\mathscr{R}$-map such that $\omega_{x}^{-1}(\operatorname{star}(i)) \subset B_{i}(1 / 4)$ for a.e. $x \in X$ and every vertex $i \in I$ in $\mathscr{N}(\mathscr{U})_{x}$. Let $\Sigma \subset \mathscr{N}(\mathscr{U})^{(k)}$ be an $\mathscr{R}$-invariant subset such that

$$
\sup _{z \in|\stackrel{s}{ }|} d(z, \operatorname{im}(\omega) \cap|s|) \geq \epsilon \text { for } s \in \Sigma .
$$

Then there exists a family of $\epsilon / 2$-projectors for $\omega$.

Proof. By compactness of $M$, we may choose a complete set $I^{\prime} \subset I$ of $\Gamma$-representatives of $I$ such that $\bigcup_{i \in I^{\prime}} B_{i}(1 / 4)$ is relatively compact. Let $K$ be the compact closure of $\bigcup_{i \in I^{\prime}} B_{i}(3 / 4)$. Let $\mathscr{F} \subset \Sigma$ be an $\mathscr{R}$-fundamental domain such that every $s \in \mathscr{F}$ has a vertex in $I^{\prime}$. Referring to the embedding $\mathscr{N}(\mathscr{U}) \subset X \times \boldsymbol{\Delta}(I)$ of Remark 2.31, $\mathscr{F}$ is a countable, disjoint union of Borel sets $X_{p} \times\left\{s_{p}\right\}, p \geq 1$, where $X_{p} \subset X$ and $s_{p} \in \boldsymbol{\Delta}(I)^{(k)}$ has at least one vertex in $I^{\prime}$. We have $\omega_{x}^{-1}(|s|) \subset K$, thus

$$
\omega_{x}(\widetilde{M}) \cap|s|=\omega_{x}(K) \cap|s|,
$$

for a.e. $x \in X$ and every $s \in \mathscr{F}_{x}$. Let $X=\bigcup_{j \in J} X_{j}$ be a countable Borel partition such that every restriction $\left.\omega\right|_{X_{j} \times K}$ is a product map. By (4.3) the set

$$
M(j, p):=\omega_{x}(\widetilde{M}) \cap\left|s_{p}\right|
$$

is constant for a.e. $x \in X_{j} \cap X_{p}$. By assumption we can pick a point $P_{j}^{p} \in\left|s_{p}^{\circ}\right|$ for every $j \in J$ and every $p \geq 1$ such that

$$
d\left(P_{j}^{p}, M(j, s)\right)>\epsilon / 2 .
$$

For $s=\left(x, s_{p}\right) \in \mathscr{F}$ with $x \in X_{j} \cap X_{p}$ define $P^{s}:=P_{j}^{p}$. Extend the definition of $P^{s}$ to $s \in \Sigma$ by equivariance. Then $\left\{P^{s}\right\}$ is admissible and a family of $\epsilon / 2$-projectors for $\omega$.

The following is a version of $[19$, Lemma D, Section 3.4] in the context of $\mathscr{R}$ spaces.

Lemma 4.10. There exists a geometric $\mathscr{R}$-map $\psi: X \times \widetilde{M} \rightarrow\left|\mathscr{N}(\mathscr{U})^{(n)}\right|$ into the $n$-skeleton such that for a.e. $x \in X$

a) $\psi$ and $\phi$ as defined in (4.2) are geometrically $\mathscr{R}$-homotopic,

b) $\psi_{x}$ has a Lipschitz constant $C=C\left(n, N_{0}\right)$ that only depends on $n$ and $N_{0}$, and

c) the $\psi_{x}$-preimage of the open star of the vertex $i \in I$ in $\left|\mathscr{N}(\mathscr{U})_{x}\right|$ is contained in $B_{i}(1 / 4)$.

Proof. Since $\mathscr{U}_{x}$ has multiplicity $\leq N_{0}$, the map $\phi$ lands in the $N_{0}$-skeleton. If $N_{0} \leq n$, setting $\psi=\phi$ will do. Let $N_{0}>n$. We inductively construct geometric $\mathscr{R}$-maps

$$
\psi^{(k)}: X \times \widetilde{M} \rightarrow\left|\mathscr{N}(\mathscr{U})^{\left(N_{0}-k\right)}\right|
$$

for $k=0,1, \ldots, N_{0}-n$ satisfying a), b) and c). Set $\psi^{(0)}=\phi$. Let $0 \leq k<N_{0}-n$. Assume $\psi^{(k)}$ is already defined; we will define $\psi^{(k+1)}$ as a map obtained from $\psi^{(k)}$ via a family of $\epsilon$-projectors $\left\{P^{s}\right\}_{s \in \mathscr{N}(\mathscr{U})^{\left(N_{0}-k\right)}}$ for $\psi^{(k)}$. If such a family $\left\{P^{s}\right\}$ exists 
with an $\epsilon>0$ that only depends on $n$ and $N_{0}$, then the resulting map $\psi^{(k+1)}$ has the desired properties by Lemma 4.8. For $s \in \mathscr{N}(\mathscr{U})_{x}^{\left(N_{0}-k\right)}$ let $M(x, s)=\psi_{x}^{(k)}(\widetilde{M}) \cap|s|$. To apply Lemma 4.9 and finish the proof, it remains to show that for a.e. $x \in X$ and every $s \in \mathscr{N}(\mathscr{U})_{x}^{\left(N_{0}-k\right)}$

$$
\epsilon_{x, s}:=\sup _{z \in|s|} d(z, M(x, s))
$$

is bounded from below in terms of $n$ and $N_{0}$. We set $\epsilon_{x, s}=\infty$ if $M(x, s)=\emptyset$. We may and will assume that $\epsilon_{x, s}<1 / 4$. Since the $n$-dimensional Hausdorff measure of $\psi_{x}^{(k)}(\widetilde{M}) \cap \mid \stackrel{s}{\mid}$ is finite by the area formula for Lipschitz maps [30, Chapter 3] whilst the $n$-dimensional Hausdorff measure of $|\stackrel{s}{\mid}|$ is infinite, $M(x, s)$ must miss a point in $\mid \stackrel{s}{s}$, thus $\epsilon_{x, s}>0$. There is a constant $D>0$ only depending on $n$ and $N_{0}$ such that there are

$$
m \geq D \epsilon_{x, s}^{-\left(N_{0}-k\right)}
$$

disjoint $\epsilon_{x, s}$ balls in $\mathscr{N}(\mathscr{U})^{\left(N_{0}-k\right)}$ with centers in $M(x, s)$ : To see this, pick a maximal packing by $\epsilon_{x, s}$-balls $B_{1}^{\prime}\left(\epsilon_{x, s}\right), \ldots, B_{m}^{\prime}\left(\epsilon_{x, s}\right)$ whose centers lie in $M(x, s)$. By (4.4), $|s|$ is covered by $B_{1}^{\prime}\left(3 \epsilon_{x, s}\right), \ldots, B_{m}^{\prime}\left(3 \epsilon_{x, s}\right)$. A volume estimate yields (4.5) for $D$ only depending on $n, N_{0}$.

Each open ball $B_{k}^{\prime}\left(\epsilon_{x, s}\right)$ lies in the union of open stars of vertices of $s$. Hence the $\psi_{x}^{(k)}$-preimages of the open balls $B_{1}^{\prime}\left(\epsilon_{x, s}\right), \ldots, B_{m}^{\prime}\left(\epsilon_{x, s}\right)$ are contained in a ball of radius 3/4. Each preimage contains a ball of radius $r=\epsilon_{x, s} C^{-1}$, where $C$ is the Lipschitz constant of $\psi^{(k)}$. By the packing inequality, $m \leq N_{0} r^{-n}=N_{0} C^{n} \epsilon_{x, s}^{-n}$. Combined with (4.5) one obtains

$$
\epsilon_{x, s} \geq\left(D N_{0}^{-1} C^{-n}\right)^{1 /\left(-n+N_{0}-k\right)}>0
$$

Proof of Theorem 4.3. Let $\psi$ be the map from Lemma 4.10. Let $\epsilon>0$ be given. For $m \geq 1$ define

$$
\begin{aligned}
\Sigma & :=\left\{s \in \mathscr{N}(\mathscr{U})^{(n)} ; \mid \stackrel{s}{\mid \not \subset \operatorname{im}}(\psi)\right\} \\
\Sigma_{m} & :=\left\{s \in \mathscr{N}(\mathscr{U})^{(n)} ; \sup _{z \in|s|} d(z, \operatorname{im}(\psi) \cap|s|) \geq 1 / m\right\} .
\end{aligned}
$$

Since $\operatorname{im}(\psi) \cap|s|$ is closed by properness of $\psi, s \in \Sigma$ implies that there is $m \geq 1$ with $s \in \Sigma_{m}$. Let $\nu_{t}$ denote the measure defined in Definition 2.5. We obtain that

$$
\lim _{m \rightarrow \infty} \nu_{t}\left(\mathscr{R} \backslash \Sigma_{m}\right)=\nu_{t}(\mathscr{R} \backslash \Sigma) .
$$

Since $\nu_{t}(\mathscr{R} \backslash \Sigma) \leq \nu_{t}(\mathscr{R} \backslash \mathscr{N}(\mathscr{U}))<\infty$ by Lemma 4.4, there is $m \geq 1$ large enough such that $\nu_{t}\left(\mathscr{R} \backslash\left(\Sigma \backslash \Sigma_{m}\right)\right)<\epsilon$. By Lemma 4.9 there is a family $\left\{P^{s}\right\}_{s \in \Sigma_{m}}$ of $1 /(2 m)$ projectors for $\psi$. Let $\psi^{\prime}$ be the map obtained from $\psi$ via $\left\{P^{s}\right\}_{s \in \Sigma_{m}}$. Define

$$
\begin{aligned}
\Sigma_{\text {hit }} & :=\left\{s \in \mathscr{N}(\mathscr{U})^{(n)} ;|\AA| \cap \operatorname{im}\left(\psi^{\prime}\right) \neq \emptyset\right\} . \\
\Sigma_{\text {full }} & :=\left\{s \in \mathscr{N}(\mathscr{U})^{(n)} ;|\AA| \subset \operatorname{im}\left(\psi^{\prime}\right)\right\}
\end{aligned}
$$

For $s \in \mathscr{N}(\mathscr{U})^{(n)}$ define

$$
D(s):=\psi^{\prime-1}(\mid \stackrel{s}{\mid)} \text {. }
$$

If $s \in \Sigma_{\text {full }}$, we have $D(s)=\psi^{-1}(|s|)$ and $\left.\psi^{\prime}\right|_{D(s)}=\left.\psi\right|_{D(s)}$. In particular, if $s \in \Sigma_{\text {full }}$, then $\left.\psi^{\prime}\right|_{D(s)}$ has the Lipschitz constant $C$ from Lemma 4.10 , and by the area formula [30, Chapter 3]

$$
\operatorname{vol}\left(\Delta^{n}\right)=\operatorname{vol}\left(\psi^{\prime}(D(s))\right) \leq C^{n} \operatorname{vol}(D(s)) .
$$


By definition of $\psi^{\prime}$ we have

$$
\Sigma_{\text {hit }} \subset \Sigma \backslash \Sigma_{m} \cup \Sigma_{\text {full }}
$$

We equip $X \times \widetilde{M}$ with the product of $\mu$ and the Riemannian measure on $\widetilde{M}$. Any $\mathscr{R}$-fundamental domain of $X \times \widetilde{M}$ has measure $\operatorname{vol}(M)$. Let $\mathscr{F}$ be a $\mathbf{S}(n+1) \times \mathscr{R}$ fundamental domain of $\Sigma_{\text {hit }}$. As usual, let $\mathscr{F}_{x}=\mathscr{F} \cap \mathscr{N}(\mathscr{U})_{x}^{(n)}$ for $x \in X$. Since the disjoint union $\bigcup_{s \in \mathscr{F}} D(s)$ is contained in an $\mathscr{R}$-fundamental domain, its measure is at most $\operatorname{vol}(M)$. Fubini's theorem yields

$$
\int_{X} \sum_{s \in \mathscr{F}_{x}} \operatorname{vol}(D(s)) d \mu(x) \leq \operatorname{vol}(M) .
$$

By Lemma 4.8 the maps $\psi_{x}^{\prime}, x \in X$, have a Lipschitz constant $C m$, so $\psi^{\prime}$ has a positive Lebesgue number (see Definition 2.25). Upon subdividing the triangulation on $M$, the map $\psi^{\prime}$ has an $\mathscr{R}$-simplicial approximation $\psi^{\prime \prime}$ by Theorem 2.26 . Note that $\operatorname{im}\left(\psi^{\prime \prime}\right)^{(n)} \subset \Sigma_{\text {hit }}$. Thus,

$$
\begin{array}{rlrl}
\sigma_{n}\left(\operatorname{im}\left(\psi^{\prime \prime}\right)\right) & =\nu_{t}\left(\mathbf{S}(n+1) \times \mathscr{R} \backslash \operatorname{im}\left(\psi^{\prime \prime}\right)\right) & \\
& \leq \nu_{t}\left(\mathbf{S}(n+1) \times \mathscr{R} \backslash \Sigma_{\text {hit }}\right) & \\
& \leq \nu_{t}\left(\mathbf{S}(n+1) \times \mathscr{R} \backslash\left(\Sigma \backslash \Sigma_{m}\right)\right)+\nu_{t}\left(\mathbf{S}(n+1) \times \mathscr{R} \backslash\left(\Sigma_{\text {hit }} \cap \Sigma_{\text {full }}\right)\right) & \text { by }(4.8) \\
& \leq \frac{\epsilon}{(n+1) !}+\int_{X} \#\left(\mathscr{F}_{x} \cap \Sigma_{\text {full }}\right) d \mu(x) & \text { by }(4.7) \\
& \leq \frac{\epsilon}{(n+1) !}+\int_{X} \sum_{s \in \mathscr{F}_{x}} C^{n} \frac{\operatorname{vol}(D(s))}{\operatorname{vol}\left(\Delta^{n}\right)} d \mu(x) & \text { by }(4.9) \\
& \leq \frac{\epsilon}{(n+1) !}+C^{n} \frac{\operatorname{vol}(M)}{\operatorname{vol}\left(\Delta^{n}\right)} .
\end{array}
$$

We apply Lemma 3.21 to $\Sigma=X \times \widetilde{M}, \Phi=\operatorname{im}\left(\psi^{\prime \prime}\right)$ and the map $\psi^{\prime \prime}$. The triangulation of $M$ naturally gives rise to a cycle $\kappa^{\prime} \in \mathrm{C}_{n}(M)$ representing the fundamental class. Let $\kappa$ be the image of $\kappa^{\prime}$ under

$$
\mathrm{C}_{n}(M)=\mathbb{Z} \otimes_{\mathbb{Z}} \mathrm{C}_{n}(\widetilde{M}) \stackrel{j_{n}}{\longrightarrow} \mathrm{L}^{\infty}(X ; \mathbb{Z}) \otimes_{\mathbb{Z} \Gamma} \mathrm{C}_{n}(\widetilde{M}) \stackrel{\lambda_{n}}{\longrightarrow} \mathscr{C}_{n}(X \times \widetilde{M}),
$$

where $j_{n}$ is the map from Remark 3.26 and $\lambda_{n}$ is the map from Lemma 3.23. Then $\kappa$ satisfies the hypothesis of Lemma 3.21. Since $\epsilon>0$ can be taken arbitrarily small in the estimate above and $\mathscr{H}_{n}(\phi)=\mathscr{H}_{n}\left(\psi^{\prime \prime}\right)$, Lemma 3.21 yields Theorem 4.3.

\subsection{Conclusion of proof.}

Proof of Theorem $A$. For $M$ as in the hypothesis and $\Gamma=\pi_{1}(M)$ pick a probability space $(X, \mu)$ as in Assumption 1.1. For example, we can always take $(X, \mu)=$ $\left(\{0,1\}^{\Gamma}, \mu_{e q}\right)$ where $\mu_{e q}$ is the infinite product of the measure $(1 / 2,1 / 2)$ on $\{0,1\}$.

By Theorems 4.1 and 4.3 there are an $\mathscr{R}$-cover $\mathscr{U}$ on $X \times \widetilde{M}$ and a geometric $\mathscr{R}$ map $\phi: X \times \widetilde{M} \rightarrow \mathscr{N}(\mathscr{U})$ such that for the fundamental class $[M]$ in $\mathscr{H}_{n}(X \times \widetilde{M})$ we have

$$
\operatorname{mass}\left(\mathscr{H}_{n}(\phi)([M])\right) \leq \operatorname{const}_{C, n} \operatorname{vol}(M),
$$

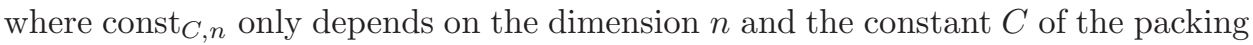
inequality. Because of Lemma $4.5 \phi$ is metrically coarse, and, by construction, $\mathscr{U}$ is uniformly bounded, and $\mathscr{N}(\mathscr{U})$ is finite-dimensional. By Lemmas 2.39 and 2.40 there is a metrically coarse, geometric $\mathscr{R}$-map $\psi: \mathscr{N}(\mathscr{U}) \rightarrow X \times \widetilde{M}$ such that the composition $X \times \widetilde{M} \stackrel{\phi}{\rightarrow} \mathscr{N}(\mathscr{U}) \stackrel{\psi}{\rightarrow} X \times \widetilde{M}$ is geometrically $\mathscr{R}$-homotopic to the identity. By Lemma 3.20 we have

$$
\operatorname{mass}([M])=\operatorname{mass}\left(\mathscr{H}_{n}(\psi \circ \phi)([M])\right) \leq \operatorname{mass}\left(\mathscr{H}_{n}(\phi)([M])\right) \leq \operatorname{const}_{C, n} \operatorname{vol}(M) .
$$


Now Theorem A follows from Theorem A.1 in the Appendix.

\section{Proofs of Theorems B And C}

5.1. $\mathscr{R}$-covers from the Ornstein-Weiss-Rokhlin lemma. The crucial ingredient in the proofs of Theorems $\mathrm{B}$ and $\mathrm{C}$ is the generalized Rokhlin-Lemma of Ornstein and Weiss.

Theorem 5.1. [31, Proposition 4 and Theorem 5; 32, Theorem 5 in II.2]

Retain Assumption 1.1. Assume that $\Gamma$ is amenable. Let $\epsilon>0$ and $\delta>0$, and let $K \subset \Gamma$ be a finite subset. Then there exist an $N \in \mathbb{N}$, independent of $\delta$ and $K, a$ sequence of $(K, \delta)$-invariant subsets $H_{1}, \ldots, H_{N}$ and Borel subsets $B_{1}, \ldots, B_{N} \subset X$ such that

a) $\left\{\gamma B_{i} ; \gamma \in H_{i}\right\}$ are $\epsilon$-disjoint for every $i \in\{1, \ldots, N\}$,

b) the sets $R_{i}:=H_{i} B_{i}$ are pairwise disjoint, and

c) $\mu\left(\bigcup_{i=1}^{N} R_{i}\right)>1-\epsilon$.

We say that Borel sets $A_{1}, \ldots, A_{n} \subset X$ are $\epsilon$-disjoint if there are pairwise disjoint Borel subsets $A_{i}^{\prime} \subset A_{i}$ with $\mu\left(A_{i}^{\prime}\right)>(1-\epsilon) \mu\left(A_{i}\right)$. For subsets $D, K \subset \Gamma$ we define

$$
\partial_{K} D:=\left\{\gamma \in D ; \exists \lambda \in\left(K \cup K^{-1}\right): \lambda \gamma \notin D\right\},
$$

and we say that $D$ is $(K, \delta)$-invariant if

$$
\frac{\# \partial_{K} D}{\# D}<\delta
$$

We need the following modified version of the previous theorem, which is nothing new. Here $\epsilon$-disjointness is replaced by disjointness but $N$ now depends on the whole setup. For convenience we include a proof.

Theorem 5.2. Retain Assumption 1.2. Assume that $\Gamma$ is amenable. Let $\epsilon>0$ and $\delta>0$, and let $K \subset \Gamma$ be a finite subset. Then there are an $N \in \mathbb{N}$ and a sequence of $(K, \delta)$-invariant subsets $H_{1}, \ldots, H_{N}$ and Borel subsets $A_{1}, \ldots, A_{N} \subset X$ such that

a) $\left\{\gamma A_{i} ; \gamma \in H_{i}\right\}$ are disjoint for every $i \in\{1, \ldots, N\}$,

b) the sets $R_{i}:=H_{i} A_{i}$ are pairwise disjoint, and

c) $\mu\left(\bigcup_{i=1}^{N} R_{i}\right)>1-\epsilon$.

Proof. Set $\delta_{0}:=\delta / 2$ and $\epsilon_{0}:=\min \left\{1 / 2, \epsilon / 2, \delta_{0} /(2 \# K+1)\right\}$. From applying Theorem 5.1 for the constants $\epsilon_{0}^{2}, \delta_{0}$ and $K$ we get $\left(K, \delta_{0}\right)$-invariant subsets $H_{1}, \ldots, H_{N_{0}} \subset$ $\Gamma$ and Borel subsets $B_{1}, \ldots, B_{N_{0}} \subset X$. For every $i \in\left\{1, \ldots, N_{0}\right\}$ and $\gamma \in H_{i}$ let $B_{i, \gamma} \subset \gamma B_{i}$ be a Borel subset such that $\mu\left(B_{i, \gamma}\right)>\left(1-\epsilon_{0}^{2}\right) \mu\left(B_{i}\right)$ and the $\left(B_{i, \gamma}\right)_{\gamma \in H_{i}}$ are pairwise disjoint. Set

$$
S_{i}:=\bigcup_{\gamma \in H_{i}}\{\gamma\} \times \gamma^{-1} B_{i, \gamma} \subset H_{i} \times B_{i} .
$$

Then the group action map $m: H_{i} \times B_{i} \rightarrow X$ is injective on $S_{i}$ and

$$
(c \times \mu)\left(S_{i}\right)>\left(1-\epsilon_{0}^{2}\right)(c \times \mu)\left(H_{i} \times B_{i}\right),
$$

where $c$ is the counting measure. For any subset $S \subset \Gamma \times X$ let

$$
c_{S}(x):=\#\{\gamma \in \Gamma ;(\gamma, x) \in S\} .
$$

Now define

$$
A_{i}:=\left\{x \in B_{i} ; c_{S_{i}}(x)>\left(1-\epsilon_{0}\right) \# H_{i}\right\} .
$$

Next we show that

$$
\mu\left(A_{i}\right)>\left(1-\epsilon_{0}\right) \mu\left(B_{i}\right) .
$$


We have the obvious estimate

$$
\begin{aligned}
(c \times \mu)\left(S_{i}\right) & \leq \mu\left(A_{i}\right) \# H_{i}+\left(1-\epsilon_{0}\right)\left(\mu\left(B_{i}\right)-\mu\left(A_{i}\right)\right) \# H_{i} \\
& =\left(1-\epsilon_{0}\right) \mu\left(B_{i}\right) \# H_{i}+\epsilon_{0} \mu\left(A_{i}\right) \# H_{i},
\end{aligned}
$$

which in combination with (5.1) yields (5.2). Let $S_{i}^{\prime}=S_{i} \cap\left(H_{i} \times A_{i}\right)$. For $x \in A_{i}$ we have $c_{S_{i}^{\prime}}(x)=c_{S_{i}}(x)>\left(1-\epsilon_{0}\right) \# H_{i}$. Together with the injectivity of $\left.m\right|_{S_{i}^{\prime}}$ and $(5.2)$ we get

$\mu\left(H_{i} A_{i}\right) \geq(c \times \mu)\left(S_{i}^{\prime}\right)>\left(1-\epsilon_{0}\right) \mu\left(A_{i}\right) \# H_{i}>\left(1-\epsilon_{0}\right)^{2} \mu\left(B_{i}\right) \# H_{i} \geq\left(1-\epsilon_{0}\right)^{2} \mu\left(H_{i} B_{i}\right)$.

Since the sets $H_{i} B_{i}$, thus the sets $R_{i}:=H_{i} A_{i}$, are pairwise disjoint,

$$
\mu\left(\bigcup_{i=1}^{N_{0}} H_{i} A_{i}\right)>\left(1-\epsilon_{0}\right)^{2} \mu\left(\bigcup_{i=1}^{N_{0}} H_{i} B_{i}\right)>\left(1-\epsilon_{0}\right)^{2}\left(1-\epsilon_{0}^{2}\right)>1-2 \epsilon_{0} .
$$

If $m\left(S_{i}^{\prime}\right) \neq H_{i} A_{i}$, we can enlarge $S_{i}^{\prime} \subset H_{i} \times A_{i}$ while keeping $\left.m\right|_{S_{i}^{\prime}}$ injective. So we may and will assume that $m\left(S_{i}^{\prime}\right)=H_{i} A_{i}$. Partition each $A_{i}$ into finitely many subsets $A_{i j}$ such that $\left(H_{i} \times\{x\}\right) \cap S_{i}^{\prime}$ is a constant set $H_{i j} \subset H_{i}$ for $x \in A_{i j}$. We obtain that

a') on each $A_{i j} \times H_{i j}$ the map $m$ is injective,

b') the sets $\left\{A_{i j} H_{i j}\right\}_{i, j}$ are pairwise disjoint, and

c') $\bigcup_{j} H_{i j} A_{i j}=H_{i} A_{i}$, thus $\mu\left(\bigcup_{i, j} H_{i j} A_{i j}\right)>1-2 \epsilon_{0} \geq 1-\epsilon$.

From $\# H_{i j} / \# H_{i}>1-\epsilon_{0}$ and the $\left(K, \delta_{0}\right)$-invariance of $H_{i}$ easily follows that $H_{i j}$ is $\left(K, \delta_{0}+(2 \# K+1) \epsilon_{0}\right)$-invariant, thus $(K, \delta)$-invariant. Now reindexing $\left(A_{i j}\right)$ and $\left(H_{i j}\right)$ as $A_{1}, \ldots, A_{N}$ and $H_{1}, \ldots, H_{N}$ gives the sets with the stated properties.

In the sequel we refer to the following setup:

Assumption 5.3. Retain the setup of Assumption 1.2, and assume that $M$ is covered by open amenable subsets $V(1), \ldots, V(m) \subset M$ such that every point in $M$ is contained in at most $n$ such subsets. We may and will assume that $V(j)$ is connected.

Write $\operatorname{incl}_{j}$ for the inclusion $V(j) \hookrightarrow M$. By hypothesis, $\Gamma(j)=\operatorname{im}\left(\pi_{1}\left(\operatorname{incl}_{j}\right)\right)$ is an amenable subgroup of $\Gamma=\pi_{1}(M)$. Let $\bar{V}(j)$ be the regular covering of $V(j)$ associated to $\operatorname{ker}\left(\pi_{1}\left(\operatorname{incl}_{j}\right)\right) \subset \pi_{1}(V(j))$. It comes with a free left action of $\Gamma(j)$. Let $p: \widetilde{M} \rightarrow M$ be the universal covering of $M$. By covering theory, we have a pullback diagram:

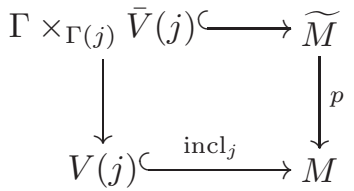

The upper map is $\Gamma$-equivariant. In other words, $p^{-1}(V(j)) \subset \widetilde{M}$ decomposes into connected components, indexed by $\Gamma / \Gamma(j)$, each of which is homeomorphic to $\bar{V}(j)$.

For every Borel map $f: S_{1} \rightarrow S_{2}$ between standard Borel spaces with countable fibers there is a Borel subset $A \subset S_{1}$ with $\left.f\right|_{A}$ injective and $f(A)=f\left(S_{1}\right)[25$, corollary 15.2 on p. 89].

In particular, there is a Borel fundamental domain $\mathscr{F}(j)$ for the $\Gamma(j)$-action on $\bar{V}(j)$, an open subset $U(j) \subset \bar{V}(j)$ and a symmetric subset $S(j) \subset \Gamma(j)$ such that

$$
\mathscr{F}(j) \subset U(j) \subset S(j) \mathscr{F}(j) .
$$

Moreover, we define

$$
\overline{\mathscr{F}}(j):=S(j) \mathscr{F}(j)
$$


Since $M$ is compact, we can take $\mathscr{F}(j)$ and $U(j)$ to be relatively compact in $\widetilde{M}$. Since the $\Gamma$-action on $\widetilde{M}$ is proper, $S(j)$ can be taken to be finite.

For every $\delta>0$ we now construct a certain $\mathscr{R}$-covering $\mathscr{U}_{\delta}$ on $X \times \widetilde{M}$. Fix $\delta>0$. For every $j \in\{1, \ldots, m\}$ we apply Theorem 5.2 to the $\Gamma(j)$-action on $X$ with the constants $\delta$ and

$$
K:=S(j)^{2}, \epsilon:=\delta
$$

and thus obtain subsets $H_{1}(j), \ldots, H_{N}(j)$ of $\Gamma(j)$ and $A_{1}(j), \ldots, A_{N}(j)$ of $X$ such that the sets $\gamma A_{i}(j)$ for $\gamma \in H_{i}(j)$ and $R_{i}(j):=H_{i}(j) A_{i}(j)$ for $i \in\{1, \ldots, N\}$ are pairwise disjoint and each $H_{i}(j)$ is $\left(S(j)^{2}, \delta\right)$-invariant. By taking the maximum over $j \in\{1, \ldots, m\}$, we can pick an $N$ that is independent of $j$. This simplifies notation a bit. Define

$$
A_{N+1}(j):=X \backslash \bigcup_{i=1}^{N} R_{i}(j) \text { and } H_{N+1}(j):=\{1\} .
$$

Then $\mu\left(A_{N+1}(j)\right)<\epsilon=\delta$. For every $j \in\{1, \ldots, m\}$ the family

$$
\mathscr{U}_{\delta}(j):=\left\{\gamma A_{i}(j) \times \gamma H_{i}(j)^{-1} U(j)\right\}_{1 \leq i \leq N+1, \gamma \in \Gamma}
$$

is an $\mathscr{R}$-cover of $X \times\left(\Gamma \times_{\Gamma(j)} \bar{V}(j)\right)=X \times p^{-1}(V(j))$.

Definition 5.4. Let $\mathscr{U}_{\delta}$ be the $\mathscr{R}$-cover of $X \times \widetilde{M}$ given by the union of $\mathscr{R}$-covers $\mathscr{U}_{\delta}(j)$ for $j \in\{1, \ldots, m\}$. Note here that a union of families is indexed by the disjoint union of their index sets.

5.2. Estimating the number of $\mathscr{R}$-cells of the nerve $\mathscr{N}\left(\mathscr{U}_{\delta}\right)$.

Theorem 5.5. Retain Assumption 5.3. If $k \geq n$, then

$$
\sigma_{k}\left(\mathscr{N}\left(\mathscr{U}_{\delta}\right)\right) \in O(\delta) \text { for } \delta \rightarrow 0 .
$$

Here the notation means that there is a constant $C$ that depends on $k, M$ and the cover $\{V(j)\}_{1 \leq j \leq m}$ but not on $\delta$ or $\mathscr{U}_{\delta}$ such that $\sigma_{k}\left(\mathscr{N}\left(\mathscr{U}_{\delta}\right)\right) \leq C \delta$ for small $\delta>0$.

Proof. Let $[m]$ denote the set $\{1,2, \ldots, m\}$. By Lemma 2.34 we have

$$
\sigma_{k}\left(\mathscr{N}\left(\mathscr{U}_{\delta}\right)\right)=\frac{1}{(k+1) !} \sum_{\substack{\left(j_{1}, \ldots, j_{k+1}\right) \in[m]^{k+1} \\\left(i_{1}, \ldots, i_{k+1}\right) \in[N+1]^{k+1}}} \sum_{\substack{\gamma_{2}, \ldots, \gamma_{k+1} \\ \text { as in }(5.8)}} \mu\left(A_{i_{1}}\left(j_{1}\right) \cap \gamma_{2} A_{i_{2}}\left(j_{2}\right) \cap \ldots \cap \gamma_{k+1} A_{i_{k+1}}\left(j_{k+1}\right)\right)
$$

where the inner sum runs over all $\gamma_{2}, \ldots, \gamma_{k+1} \in \Gamma$ such that

$$
\left\{\begin{array}{l}
H_{i_{1}}\left(j_{1}\right)^{-1} U\left(j_{1}\right) \cap \gamma_{2} H_{i_{2}}\left(j_{2}\right)^{-1} U\left(j_{2}\right) \cap \ldots \cap \gamma_{k} H_{i_{k}}\left(j_{k}\right)^{-1} U\left(j_{k}\right) \neq \emptyset \\
\left(\gamma_{i_{r}}, i_{r}, j_{r}\right) \neq\left(\gamma_{i_{s}}, i_{s}, j_{s}\right) \text { for } 1 \leq r \neq s \leq k+1 \text { and } \gamma_{1}=1
\end{array}\right.
$$

Define

$$
\Sigma\left(j_{1}, \ldots, j_{k}\right):=\sum_{\left(i_{1}, \ldots, i_{k}\right) \in[N+1]^{k}} \sum_{\begin{array}{c}
\gamma_{2}, \ldots, \gamma_{k}, \\
h_{1}, \ldots, h_{k} \\
\text { as in }(5.9)
\end{array}} \mu\left(A_{i_{1}}\left(j_{1}\right) \cap \gamma_{2} A_{i_{2}}\left(j_{2}\right) \ldots \cap \gamma_{k} A_{i_{k}}\left(j_{k}\right)\right)
$$

where the inner sum runs over all $\gamma_{2}, \ldots, \gamma_{k} \in \Gamma$ and $h_{1}, \ldots, h_{k} \in \Gamma$ such that $h_{l} \in H_{i_{l}}\left(j_{l}\right)$ and

$$
\left\{\begin{array}{l}
h_{1}^{-1} \overline{\mathscr{F}}\left(j_{1}\right) \cap \gamma_{2} h_{2}^{-1} \overline{\mathscr{F}}\left(j_{2}\right) \cap \ldots \cap \gamma_{k} h_{k}^{-1} \overline{\mathscr{F}}\left(j_{k}\right) \neq \emptyset \\
\left(\gamma_{i_{r}}, i_{r}, j_{r}\right) \neq\left(\gamma_{i_{s}}, i_{s}, j_{s}\right) \text { for } 1 \leq r \neq s \leq k \text { and } \gamma_{1}=1
\end{array}\right.
$$


The sum $\sum \Sigma\left(j_{1}, \ldots, j_{k+1}\right)$ over all $\left(j_{1}, \ldots, j_{k+1}\right) \in[m]^{k+1}$ clearly dominates $\sigma_{k}\left(\mathscr{N}\left(\mathscr{U}_{\delta}\right)\right)$ because of (5.5) and (5.6). To prove the claim, it thus suffices to prove that

$$
\Sigma\left(j_{1}, \ldots, j_{k+1}\right) \in O(\delta) \text { for } \delta \rightarrow 0 .
$$

for every $\left(j_{1}, \ldots, j_{k+1}\right) \in[m]^{n+1}$ provided $k \geq n$. We need the following two lemmas to continue.

Lemma 5.6. Let $k \geq 1$. There is a constant $C>0$ that does not depend on $\delta$ and $\mathscr{U}_{\delta}$ such that

$$
\Sigma\left(j_{1}, \ldots, j_{k+1}\right) \leq C \cdot \Sigma\left(j_{1}, \ldots, j_{k}\right) .
$$

Proof of lemma. There is a finite set $F \subset \Gamma$ such that for all $j, j^{\prime} \in[m]$

$$
\gamma \overline{\mathscr{F}}(j) \cap \overline{\mathscr{F}}\left(j^{\prime}\right) \neq \emptyset \Rightarrow \gamma \in F .
$$

This is clear since $\Gamma$ acts properly on $\widetilde{M}$ and each $\overline{\mathscr{F}}(j)$ is relatively compact. Now $\Sigma\left(j_{1}, \ldots, j_{k+1}\right)$ is bounded by

$$
\sum_{\substack{\left(i_{1}, \ldots, i_{k}\right) \in[N+1]^{k} \\ \gamma_{l}, h_{l} \text { as in }(5.11)}} \sum_{\substack{f \in F \\ i_{k+1} \in[N+1] \\ h \in H_{i_{k+1}}}} \mu\left(A_{i_{1}}\left(j_{1}\right) \cap \ldots \cap \gamma_{k} A_{i_{k}}\left(j_{k}\right) \cap \gamma_{k} h_{k}^{-1} f h A_{i_{k+1}}\left(j_{k+1}\right)\right) .
$$

In the first sum we sum over all $k$-tuples $\left(1, \gamma_{2}, \ldots, \gamma_{k}\right) \in \Gamma^{k+1}$ and $\left(h_{1}, \ldots, h_{k}\right) \in$ $H_{i_{1}}\left(j_{1}\right) \times \ldots \times H_{i_{k}}\left(j_{k}\right)$ that satisfy

$$
\left\{\begin{array}{l}
h_{1}^{-1} \overline{\mathscr{F}}\left(j_{1}\right) \cap \gamma_{2} h_{2}^{-1} \overline{\mathscr{F}}\left(j_{2}\right) \cap \ldots \cap \gamma_{k} h_{k}^{-1} \overline{\mathscr{F}}\left(j_{k}\right) \neq \emptyset \\
\left(\gamma_{i_{r}}, i_{r}, j_{r}\right) \neq\left(\gamma_{i_{s}}, i_{s}, j_{s}\right) \text { for } 1 \leq r \neq s \leq k \text { and } \gamma_{1}=1 .
\end{array}\right.
$$

The family $\left\{\gamma_{k} h_{k}^{-1} f h A_{i_{k+1}}\left(j_{k+1}\right)\right\}_{i_{k+1} \in[N+1], h \in H_{i_{k+1}}}$ (for fixed $\left.f, h_{k}, \gamma_{k}\right)$ is a Borel partition of $X$. Hence the claim is true for $C=\# F$.

Lemma 5.7. For $j \in[N+1]$, we have $\Sigma(j, j) \in O(\delta)$ for $\delta \rightarrow 0$.

Proof of lemma. For $\bar{V}(j) \cap \gamma \bar{V}(j) \neq \emptyset$ it is necessary that $\gamma \in \Gamma(j)$ (see diagram (5.4)). If $h^{-1} \overline{\mathscr{F}}(j) \cap \gamma h^{\prime-1} \mathscr{\mathscr { F }}(j) \neq \emptyset$ for $h, h^{\prime} \in \Gamma(j)$ then $\gamma \in h^{-1} S(j)^{2} h^{\prime}$ because $\mathscr{F}(j)$ is a $\Gamma(j)$-fundamental domain and because of (5.6). Thus

$$
\begin{aligned}
\Sigma(j, j) & \leq \sum_{\substack{i_{1}, i_{2} \in[N+1] \\
h_{1} \in H_{i_{1}}(j)}} \sum_{h_{2} \in H_{i_{2}}(j)} \sum_{\substack{s \in S(j)^{2} \\
h_{1} \neq s h_{2} \text { if } i_{1}=i_{2}}} \mu\left(A_{i_{1}}(j) \cap h_{1}^{-1} s h_{2} A_{i_{2}}(j)\right) \\
& =\sum_{\substack{i_{1}, i_{2} \in[N+1] \\
h_{1} \in H_{i_{1}}(j)}} \sum_{h_{2} \in H_{i_{2}}(j)} \sum_{\substack{s \in S(j)^{2} \\
h_{1} \neq s h_{2} \text { if } i_{1}=i_{2}}} \mu\left(h_{1} A_{i_{1}}(j) \cap s h_{2} A_{i_{2}}(j)\right)
\end{aligned}
$$

Notice that $\mu\left(h_{1} A_{i_{1}}(j) \cap s h_{2} A_{i_{2}}(j)\right)=0$ if $s h_{2} \in H_{i_{2}}(j)$. Thus,

$$
\begin{aligned}
\Sigma(j, j) & \leq \sum_{\substack{i_{1}, i_{2} \in[N+1] \\
h_{1} \in H_{i_{1}}(j)}} \sum_{h_{2} \in \partial_{S(j)^{2}} H_{i_{2}}(j)} \sum_{s \in S(j)^{2}} \mu\left(h_{1} A_{i_{1}}(j) \cap s h_{2} A_{i_{2}}(j)\right) \\
& =\sum_{i_{2} \in[N+1]} \sum_{h_{2} \in \partial_{S(j)^{2}} H_{i_{2}}(j)} \sum_{s \in S(j)^{2}} \mu\left(s h_{2} A_{i_{2}}(j)\right) \\
& =\sum_{i_{2} \in[N+1]} \sum_{h_{2} \in \partial_{S(j)^{2}} H_{i_{2}}(j)} \sum_{s \in S(j)^{2}} \mu\left(H_{i_{2}}(j) A_{i_{2}}(j)\right) / \# H_{i_{2}}(j) \\
& \leq \# S(j)^{2} \delta \sum_{i_{2} \in[N+1]} \mu\left(H_{i_{2}}(j) A_{i_{2}}(j)\right) \\
& =\# S(j)^{2} \delta .
\end{aligned}
$$


Continuation of proof of Theorem 5.5. It remains to verify (5.10). If $k \geq n$, then $\Sigma\left(j_{1}, \ldots, j_{k+1}\right)=0$ unless two of the entries of $\left(j_{1}, \ldots, j_{k+1}\right)$ are equal because of (5.9) and the fact that $\{V(j)\}_{1 \leq j \leq m}$ has multiplicity at most $n$.

We can assume that $j=j_{1}=j_{2}$ without loss of generality. Then by repeated application of Lemma 5.6 , we obtain that $\Sigma\left(j_{1}, \ldots, j_{k+1}\right) \leq C^{k-1} \Sigma(j, j)$ where $C$ is the constant from Lemma 5.6, and Lemma 5.7 finally yields (5.10).

5.3. Conclusion of proofs. Let $M$ be as in the hypothesis of Theorem B or Theorem C. For $\Gamma=\pi_{1}(M)$ let $(X, \mu)$ be as in Assumption 1.1. For example, take the Bernoulli space $(X, \mu)=\left(\{0,1\}^{\Gamma}, \mu_{e q}\right)$ where $\mu_{e q}$ is the infinite product of the measure $(1 / 2,1 / 2)$ on $\{0,1\}$. Let $\mathscr{U}_{\delta}$ be the $\mathscr{R}$-cover on $X \times \widetilde{M}$ constructed in Subsection 5.1. It is immediate from the construction that $\mathscr{U}_{\delta}$ is uniformly bounded and $\mathscr{N}\left(\mathscr{U}_{\delta}\right)$ is finite-dimensional.

By Lemmas 2.35 and 2.39 there exists an $\mathscr{R}$-simplicial (thus, metrically coarse) map

$$
\phi: X \times \widetilde{M} \rightarrow \mathscr{N}\left(\mathscr{U}_{\delta}\right)
$$

(after subdividing the domain). By Lemma 2.40 there is a metrically coarse geometric $\mathscr{R}$-map

$$
\psi: \mathscr{N}\left(\mathscr{U}_{\delta}\right) \rightarrow X \times \widetilde{M}
$$

such that there is a geometric $\mathscr{R}$-homotopy between $\mathrm{id}_{X \times \widetilde{M}}$ and $\psi \circ \phi$.

End of proof of Theorem B. The triangulation of $M$ naturally gives rise to a cycle $\kappa^{\prime} \in \mathrm{C}_{n}(M)$. Let $\kappa$ be the image of $\kappa^{\prime}$ under

$$
\mathrm{C}(M)=\mathbb{Z} \otimes_{\mathbb{Z} \Gamma} \mathrm{C}(\widetilde{M}) \stackrel{j_{n}}{\longrightarrow} \mathrm{L}^{\infty}(X ; \mathbb{Z}) \otimes_{\mathbb{Z} \Gamma} \mathrm{C}(\widetilde{M}) \stackrel{\lambda_{n}}{\longrightarrow} \mathscr{C}_{n}(X \times \widetilde{M}),
$$

where $j_{n}$ and $\lambda_{n}$ are the maps from Remark 3.26 and Lemma 3.23, respectively. Then $\kappa$ represents the fundamental class $[M]$ in $\mathscr{H}_{n}(X \times \widetilde{M})$, and $\kappa$ and $\phi$ satisfy the hypothesis of Lemma 3.21.

By Lemma 3.21 and Theorem 5.5 we have

$$
\operatorname{mass}\left(\mathscr{H}_{n}(\phi)([M])\right) \leq(n+1) !^{2} \cdot \sigma_{n}\left(\mathscr{N}\left(\mathscr{U}_{\delta}\right)\right) \in O(\delta) .
$$

Lemma 3.20 yields

$$
\operatorname{mass}([M])=\operatorname{mass}(\mathscr{H}(\psi \circ \phi)([M])) \leq \operatorname{mass}\left(\mathscr{H}_{n}(\phi)([M])\right) \in O(\delta) .
$$

Now Theorem B follows from Theorem A.1 and by letting $\delta \rightarrow 0$.

Before we come to the proof of Theorem C, we need the following lemma that we extract from Gaboriau's theory of $L^{2}$-Betti numbers $b_{k}^{(2)}(\Sigma)$ for arbitrary $\mathscr{R}$ simplicial complexes [17, Section 4.3].

Lemma 5.8. Let $\Sigma, \Psi$ be $\mathscr{R}$-simplicial complexes such that $\Sigma_{x}$ is contractible for a.e. $x \in X$. Let $\phi: \Sigma \rightarrow \Psi$ be an $\mathscr{R}$-simplicial map. Then

$$
b_{k}^{(2)}(\Sigma) \leq b_{k}^{(2)}(\Psi) \text { for all } k \geq 0
$$

Proof. The proof is essentially the same as the one of [17, Théorème 3.13]; there the hypothesis of the existence of $\phi$ is replaced by an assumption on the connectivity of $\Psi$. We only indicate the necessary modifications: The map $\phi$ induces a champ borélien équivariant $t_{x}^{*}: \mathrm{C}_{*}\left(\Sigma_{x}\right) \rightarrow \mathrm{C}_{*}\left(\Psi_{x}\right)$ in the sense of [17, Définition 3.4]. For the $t_{x}^{*}$ we can find $s_{x}^{*}, r_{x}^{*}$ as in the diagram of [17, Lemme 4.6]. The proof [17, Lemme 4.6] almost literally translates to our situation. Now the assertion follows from [17, Théorème 4.8] 
End of proof of Theorem $C$. Applying Lemma 5.8 to $\Sigma=X \times \widetilde{M}, \Psi=\mathscr{N}\left(\mathscr{U}_{\delta}\right)$ and the map in (5.12), we get the estimate

$$
b_{k}^{(2)}(\widetilde{M})=b_{k}^{(2)}(X \times \widetilde{M}) \leq b_{k}^{(2)}\left(\mathscr{N}\left(\mathscr{U}_{\delta}\right)\right)
$$

for all $k \geq 0$. We have $b_{k}^{(2)}\left(\mathscr{N}\left(\mathscr{U}_{\delta}\right)\right) \leq \sigma_{k}\left(\mathscr{N}\left(\mathscr{U}_{\delta}\right)\right)$ [17, Proposition $\left.3.2(3)\right]$. Since $\sigma_{k}\left(\mathscr{N}\left(\mathscr{U}_{\delta}\right)\right) \in O(\delta)$ for $k \geq n$ by Theorem 5.5, the assertion follows for $\delta \rightarrow 0$.

\section{Appendix A. L²-Betti numbers AND The Support MASS OF THE FUNDAMENTAL CLASS}

Throughout, we retain the setting of Assumption 1.3. This appendix is devoted to Theorem A.1 below. A statement of this kind was posed in Gromov's book [23, 5.38 e) on p. 304] as an exercise. A proof of Theorem A.1 is given in the doctoral thesis [35]. The proof we present at the end of this Appendix is basically that of [35] and only differs in the discussion of equivariant Poincaré duality.

Theorem A.1. Let $[M]$ be the fundamental class in $\mathscr{H}_{n}(X \times \widetilde{M})$ (cf. Remark 3.26). Then

$$
b_{p}^{(2)}(\widetilde{M}) \leq \operatorname{mass}([M]) \text { for all } p \geq 0 .
$$

The proof of Theorem A.1 is essentially an application of equivariant Poincaré duality. The presentation of the latter in [28] very much fits for our purposes. First we discuss the necessary algebraic objects.

Definition. The twisted group ring $\mathrm{L}^{\infty}(X ; \mathbb{C}) \rtimes \Gamma$ is, as an $\mathrm{L}^{\infty}(X ; \mathbb{C})$-module, free with basis $\Gamma$. Its ring multiplication extends uniquely that of $\mathrm{L}^{\infty}(X ; \mathbb{C})$ and $\mathbb{C} \Gamma$ such that the commutation rule $f \gamma=\gamma f^{\gamma}$ holds. Recall that $\Gamma$ acts on $\mathrm{L}^{\infty}(X ; \mathbb{Z})$ from the right by $f^{\gamma}(x)=(f \gamma)(x)=f(\gamma x)$. There is a ring involution on $\mathrm{L}^{\infty}(X ; \mathbb{C}) \rtimes \Gamma$ given by $\bar{\gamma}=\gamma^{-1}$ where $\bar{f}(x)=f \overline{(x)}$ is complex conjugation.

The twisted group ring $\mathrm{L}^{\infty}(X ; \mathbb{C}) \rtimes \Gamma$ is equipped with the trace

$$
\operatorname{tr}: \mathrm{L}^{\infty}(X ; \mathbb{C}) \rtimes \Gamma \rightarrow \mathbb{C}, \operatorname{tr}\left(\sum_{i=1}^{k} f_{k} \gamma_{k}\right)=\int_{X} f_{1}(x) d \mu(x)
$$

where $\gamma_{1}=1 \in \Gamma, \gamma_{2}, \ldots, \gamma_{k} \in \Gamma \backslash\{1\}$ and $f_{1}, \ldots, f_{k} \in \mathrm{L}^{\infty}(X ; \mathbb{C})$. The corresponding GNS-construction defines a von Neumann algebra $L(X, \Gamma)$ with a finite trace, which is commonly referred to as the group measure space construction. The group von Neumann algebra $L(\Gamma)$ is contained in $L(X, \Gamma)$.

Let $\mathrm{C}_{*}, \mathrm{D}_{*}$ denote $\mathbb{Z} \Gamma$-chain complexes. Let $\mathrm{C}^{-*}$ be the chain complex whose $p$-th chain module is $\operatorname{hom}_{\mathbb{Z} \Gamma}\left(C_{-p}, \mathbb{Z} \Gamma\right)$ with the induced differential. The minus sign causes $\mathrm{C}^{-*}$ to be chain complex rather than a cochain complex. Naturally, $\mathrm{C}^{-*}$ is a right $\mathbb{Z} \Gamma$-module but every right module over a ring with involution can be viewed as a left module. Furthermore, $\operatorname{hom}_{\mathbb{Z} \Gamma}\left(\mathrm{C}^{-*}, \mathrm{D}_{*}\right)$ is the hom-complex; it is again a chain complex whose $p$-th chain group consists of degree $p$ chain maps $\mathrm{C}^{-*} \rightarrow \mathrm{D}_{*}$. Its $p$-th homology consists of homotopy classes of such.

For brevity, let us write $\mathrm{L}^{\infty}$ instead of $\mathrm{L}^{\infty}(X ; \mathbb{Z})$ in the sequel. We have the following commutative square of chain homomorphisms

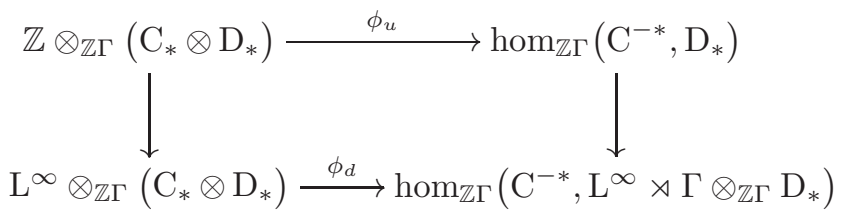




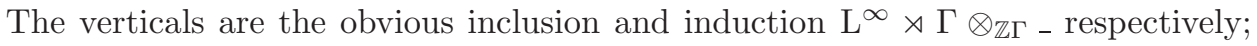
note here that the lower right corner is canonically isomorphic to

$$
\operatorname{hom}_{\mathrm{L} \rtimes}\left(\mathrm{L}^{\infty} \rtimes \Gamma \otimes_{\mathbb{Z} \Gamma} \mathrm{C}^{-*}, \mathrm{~L}^{\infty} \rtimes \Gamma \otimes_{\mathbb{Z} \Gamma} \mathrm{D}_{*}\right) .
$$

The upper homomorphism is given by $\phi_{u}(1 \otimes x \otimes y)(g)=\overline{g(x)} y$ for $g \in \mathrm{C}^{-*}$; the lower homomorphism is defined by $\phi_{d}(f \otimes x \otimes y)(g)=\overline{f g(x)} \otimes y$.

Let $A W_{*}: \mathrm{C}_{*}(\widetilde{M} \times \widetilde{M}) \rightarrow \mathrm{C}_{*}(\widetilde{M}) \otimes_{\mathbb{Z}} \mathrm{C}_{*}(\widetilde{M})$ be the Alexander-Whitney map, and let $\Delta_{*}: \mathrm{C}_{*}(\widetilde{M}) \rightarrow \mathrm{C}_{*}(\widetilde{M} \times \widetilde{M})$ be the chain map coming from the diagonal inclusion. Both maps are $\mathbb{Z} \Gamma$-equivariant with the appropriate diagonal $\Gamma$-action on the target.

We obtain the following commutative square:

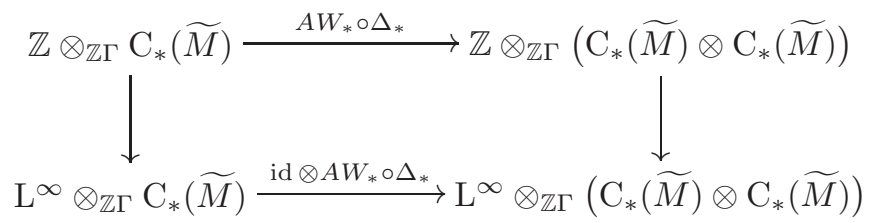

If we set $\mathrm{C}_{*}=\mathrm{C}_{*}(\widetilde{M})$, concatenate both squares above and take the $n$-th homology, we obtain the following diagram.

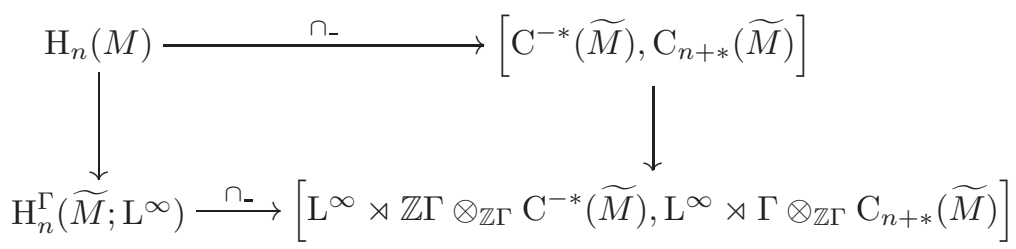

The square brackets denote homotopy classes of chain maps. A representative of the image of $z$ under one of the horizontal maps is a chain map that is called the cap product with $z$.

Now consider an element $z \in H_{n}(M)$ and its image $z^{\prime}$ in $\mathrm{H}_{n}^{\Gamma}\left(\widetilde{M} ; \mathrm{L}^{\infty}\right)$. The cap product with $z^{\prime}$ defines, after induction with $L(X, \Gamma) \otimes_{\mathrm{L}^{\infty} \rtimes \Gamma-}$, an $L(X, \Gamma)$-homomorphism (up to homotopy)

$$
-\cap z^{\prime}: L(X, \Gamma) \otimes_{\mathbb{Z} \Gamma} \mathrm{C}^{-*}(\widetilde{M}) \longrightarrow L(X, \Gamma) \otimes_{\mathbb{Z} \Gamma} \mathrm{C}_{n+*}(\widetilde{M}) .
$$

According to equivariant Poincaré duality, the map (A.1) is a $\mathbb{Z} \Gamma$-homotopy equivalence if $z \in H_{n}(M)$ is the fundamental class of $M$ (already before induction with $L(X, \Gamma) \otimes_{\left.L^{\infty} \rtimes \Gamma_{-}\right)}$. Let us take a closer look at this map. Suppose $z^{\prime}$ is represented by the cycle $\sum_{k=1}^{m} f_{k} \otimes \sigma_{k}$ with $f_{k} \in \mathrm{L}^{\infty}, \sigma_{k}: \Delta^{n} \rightarrow \widetilde{M}$. With the standard formula for the Alexander-Whitney map, we can unravel the definition of (A.1) and see that it sends the element $1 \otimes g \in L(X, \Gamma) \otimes_{\mathbb{Z} \Gamma} \mathrm{C}^{j}(\widetilde{M})$ to

$$
\sum_{k=1}^{m} \overline{\left.f_{k} g\left(\sigma_{k}\right\rfloor_{j}\right)} \otimes \sigma_{k}\left\lfloor_{n-j} \in L(X, \Gamma) \otimes_{\mathbb{Z} \Gamma} \mathrm{C}_{n-j}(\widetilde{M}) .\right.
$$

Here $\sigma\rfloor_{j}$ and $\sigma\left\lfloor n-j_{n}\right.$ denote the front $j$-face and the back $(n-j)$-face of $\sigma$ respectively. It follows that the kernel $\operatorname{ker}\left(e v_{j}\right)$ of

$$
\begin{gathered}
e v_{j}: L(X, \Gamma) \otimes_{\mathbb{Z} \Gamma} \mathrm{C}^{j}(\widetilde{M}) \rightarrow \bigoplus_{k=1}^{m} L(X, \Gamma) \chi_{\text {supp } f_{k}}, \\
x \otimes g \mapsto\left(x \overline{\left.f_{k} g\left(\sigma_{k}\right\rfloor_{j}\right)}=x \overline{\left.g\left(\sigma_{k}\right\rfloor_{j}\right)} f_{k}\right)_{k}
\end{gathered}
$$

is contained in $\operatorname{ker}\left(\_\cap z^{\prime}\right)$; here $\chi_{\text {supp } f_{k}}$ is the characteristic function of the support of $f_{k}$. 
To follow the proof of Theorem A.1, the reader must be aware of the following facts.

a) There is a dimension for every (algebraic) module $M$ over a von Neumann algebra $\mathcal{A}$ with a finite $\operatorname{trace}_{\operatorname{tr}}: \mathcal{A} \rightarrow \mathbb{C}$, denoted by $\operatorname{dim}_{\mathcal{A}}(M) \in[0, \infty]$.

b) The dimension $\operatorname{dim}_{\mathcal{A}}$ is additive for short exact sequences of $\mathcal{A}$-modules.

c) For an idempotent $p \in \mathcal{A}, \operatorname{dim}_{\mathcal{A}}(\mathcal{A} p)=\operatorname{tr}_{\mathcal{A}}(p)$.

d) $b_{i}^{(2)}(\widetilde{M})=\operatorname{dim}_{L(\Gamma)}\left(\mathrm{H}_{i}^{\Gamma}(\widetilde{M} ; L(\Gamma))\right)$

e) $\operatorname{dim}_{L(\Gamma)}\left(\mathrm{H}_{i}^{\Gamma}(\widetilde{M} ; L(\Gamma))\right)=\operatorname{dim}_{L(X, \Gamma)}\left(\mathrm{H}_{i}^{\Gamma}(\widetilde{M} ; L(X, \Gamma))\right)$.

The items a)-c) belong to the core of Lück's dimension theory for a von Neumann algebra with a finite trace $[27$, chapter 6$]$; d) is a consequence of $[33$, Theorem 2.6 and Theorem 4.3]. See also [34, Theorem 6.8].

Proof of Theorem A.1. Let $\epsilon>0$. By Theorem 3.25 the fundamental class $z^{\prime}$ in $\mathrm{H}_{n}^{\Gamma}\left(\widetilde{M} ; \mathrm{L}^{\infty}\right)$ can be represented by a cycle

$$
\sum_{k=1}^{m} f_{k} \otimes \sigma_{k} \in \mathrm{L}^{\infty} \otimes_{\mathbb{Z} \Gamma} \mathrm{C}_{n}(\widetilde{M})
$$

such that

$$
\sum_{k=1}^{m} \mu\left(\operatorname{supp}\left(f_{k}\right)\right)<\operatorname{mass}([M])+\epsilon
$$

where $[M]$ denotes the fundamental class in $\mathscr{H}_{n}(X \times \widetilde{M})$.

Let $\delta^{j}$ denote the $j$-th differential of $L(X, \Gamma) \otimes_{\mathbb{Z} \Gamma} \mathrm{C}^{*}(\widetilde{M})$. There is always a projection $\operatorname{ker}\left(\delta^{j}\right) \rightarrow \mathrm{H}_{\Gamma}^{j}(\widetilde{M} ; L(X, \Gamma))$. If we compose it with the homology homomorphism induced by (A.1), we obtain a map

$$
\operatorname{ker}\left(\delta^{j}\right) \rightarrow \mathrm{H}_{\Gamma}^{j}(\widetilde{M} ; L(X, \Gamma)) \stackrel{\cong}{\longrightarrow} \mathrm{H}_{n-j}^{\Gamma}(\widetilde{M} ; L(X, \Gamma))
$$

that factors over $\operatorname{ker}\left(e v_{j}\right)$ since $\operatorname{ker}\left(e v_{j}\right)$ is contained in $\operatorname{ker}\left(-\cap z^{\prime}\right)$. Thus

$$
b_{n-j}^{(2)}(\widetilde{M})=\operatorname{dim}_{L(X, \Gamma)}\left(\mathrm{H}_{n-j}^{\Gamma}(\widetilde{M}, L(X, \Gamma))\right) \leq \operatorname{dim}_{L(X, \Gamma)}\left(\operatorname{ker}\left(\delta^{j}\right) / \operatorname{ker}\left(e v_{j}\right)\right) .
$$

Since $\operatorname{ker}\left(\delta^{j}\right) / \operatorname{ker}\left(e v_{j}\right)$ injects into

$$
\bigoplus_{k=1}^{m} L(X, \Gamma) \chi_{\operatorname{supp} f_{k}}
$$

we conclude with (A.2) that

$$
b_{n-j}^{(2)}(\widetilde{M}) \leq \sum_{k=1}^{m} \mu\left(\operatorname{supp}\left(f_{k}\right)\right)<\operatorname{mass}([M])+\epsilon .
$$

\section{REFERENCES}

[1] Michael F. Atiyah, Elliptic operators, discrete groups and von Neumann algebras, Colloque "Analyse et Topologie" en l'Honneur de Henri Cartan (Orsay, 1974), Soc. Math. France, Paris, 1976, pp. 43-72. Astérisque, No. 32-33.

[2] Arthur Bartels and David Rosenthal, On the K-theory of groups with finite asymptotic dimension (2006), available at arXiv:math.KT/0605088.

[3] G. Bell and A. Dranishnikov, On asymptotic dimension of groups acting on trees, Geom. Dedicata 103 (2004), 89-101.

[4] Marcel Berger, A panoramic view of Riemannian geometry, Springer-Verlag, Berlin, 2003.

[5] Armand Borel, The $L^{2}$-cohomology of negatively curved Riemannian symmetric spaces, Ann. Acad. Sci. Fenn. Ser. A I Math. 10 (1985), 95-105.

[6] Glen E. Bredon, Topology and geometry, Graduate Texts in Mathematics, vol. 139, SpringerVerlag, New York, 1993.

[7] Jeff Cheeger and Mikhael Gromov, $L_{2}$-cohomology and group cohomology, Topology 25 (1986), no. 2, 189-215. 
[8] Jeff Cheeger and Tobias H. Colding, Lower bounds on Ricci curvature and the almost rigidity of warped products, Ann. of Math. (2) 144 (1996), no. 1, 189-237.

[9] Donald L. Cohn, Measure theory, Birkhäuser Boston, Mass., 1980.

[10] Alain Connes, Sur la théorie non commutative de l'intégration, Algèbres d'opérateurs (Sém., Les Plans-sur-Bex, 1978), Lecture Notes in Math., vol. 725, Springer, Berlin, 1979, pp. 19-143 (French).

[11] Jozef Dodziuk, de Rham-Hodge theory for $L^{2}$-cohomology of infinite coverings, Topology 16 (1977), no. 2, 157-165.

[12] Michael Farber, von Neumann categories and extended $L^{2}$-cohomology, K-Theory 15 (1998), no. 4, 347-405.

[13] Jacob Feldman and Calvin C. Moore, Ergodic equivalence relations, cohomology, and von Neumann algebras. I, Trans. Amer. Math. Soc. 234 (1977), no. 2, 289-324.

[14] Ergodic equivalence relations, cohomology, and von Neumann algebras. II, Trans. Amer. Math. Soc. 234 (1977), no. 2, 325-359.

[15] Alex Furman, Gromov's measure equivalence and rigidity of higher rank lattices, Ann. of Math. (2) 150 (1999), no. 3, 1059-1081.

[16] _ Orbit equivalence rigidity, Ann. of Math. (2) 150 (1999), no. 3, 1083-1108.

[17] Damien Gaboriau, Invariants $l^{2}$ de relations d'équivalence et de groupes, Publ. Math. Inst. Hautes Études Sci. (2002), no. 95, 93-150 (French).

[18] Sylvestre Gallot, Dominique Hulin, and Jacques Lafontaine, Riemannian geometry, 3rd ed., Universitext, Springer-Verlag, Berlin, 2004.

[19] Mikhael Gromov, Volume and bounded cohomology, Inst. Hautes Études Sci. Publ. Math. (1982), no. 56, 5-99 (1983).

[20] , Foliated Plateau problem. I. Minimal varieties, Geom. Funct. Anal. 1 (1991), no. 1, 14-79.

[21] _ Kähler hyperbolicity and $L_{2}$-Hodge theory, J. Differential Geom. 33 (1991), no. 1, $263-292$.

[22] - Asymptotic invariants of infinite groups, Geometric group theory, Vol. 2 (Sussex, 1991), London Math. Soc. Lecture Note Ser., vol. 182, Cambridge Univ. Press, Cambridge, 1993, pp. 1-295.

[23] - Metric structures for Riemannian and non-Riemannian spaces, Progress in Mathematics, vol. 152, Birkhäuser Boston Inc., Boston, MA, 1999.

[24] Nigel Higson and John Roe, Amenable group actions and the Novikov conjecture, J. Reine Angew. Math. 519 (2000), 143-153.

[25] Alexander S. Kechris, Classical descriptive set theory, Graduate Texts in Mathematics, vol. 156, Springer-Verlag, New York, 1995.

[26] Wolfgang Lück, Dimension theory of arbitrary modules over finite von Neumann algebras and $L^{2}$-Betti numbers. I. Foundations, J. Reine Angew. Math. 495 (1998), 135-162.

[27] — $L^{2}$-invariants: theory and applications to geometry and $K$-theory, Ergebnisse der Mathematik und ihrer Grenzgebiete. 3. Folge. A Series of Modern Surveys in Mathematics [Results in Mathematics and Related Areas. 3rd Series. A Series of Modern Surveys in Mathematics], vol. 44, Springer-Verlag, Berlin, 2002

[28] A basic introduction to surgery theory, Topology of high-dimensional manifolds, No. 1, 2 (Trieste, 2001), ICTP Lect. Notes, vol. 9, Abdus Salam Int. Cent. Theoret. Phys., Trieste, 2002, pp. 1-224.

[29] Calvin C. Moore and Claude Schochet, Global analysis on foliated spaces, Mathematical Sciences Research Institute Publications, vol. 9, Springer-Verlag, New York, 1988. With appendices by S. Hurder, Moore, Schochet and Robert J. Zimmer.

[30] Frank Morgan, Geometric measure theory, Academic Press Inc., San Diego, CA, 2000.

[31] Donald S. Ornstein and Benjamin Weiss, Ergodic theory of amenable group actions. I. The Rohlin lemma, Bull. Amer. Math. Soc. (N.S.) 2 (1980), no. 1, 161-164.

[32] Entropy and isomorphism theorems for actions of amenable groups, J. Analyse Math. 48 (1987), 1-141.

[33] Roman Sauer, $L^{2}$-Betti numbers of discrete measured groupoids, Internat. J. Algebra Comput. 15 (2005), no. 5-6, 1169-1188.

[34] _ Homological invariants and quasi-isometry, Geom. Funct. Anal. 16 (2006), no. 2.

[35] Marco Schmidt, $L^{2}$-Betti numbers of $\mathscr{R}$-spaces and the Integral Foliated Simplicial Volume, Universität Münster, 2005. doctoral thesis.

[36] Yehuda Shalom, Measurable group theory, European Congress of Mathematics, Eur. Math. Soc., Zürich, 2005, pp. 391-423.

[37] Edwin H. Spanier, Algebraic topology, Springer-Verlag, New York, 1981. Corrected reprint.

[38] V. S. Varadarajan, Groups of automorphisms of Borel spaces, Trans. Amer. Math. Soc. 109 (1963), 191-220. 
Department of Mathematics, University of Chicago, 5734 S. University Avenue, Chicago, IL 60637

E-mail address: romansauer@member.ams.org

$U R L$ : www.romansauer.de 